\title{
Evidence for wind and accretion in the Herbig Be star HD 100546 from FUSE observations
}

\author{
M. Deleuil ${ }^{1,2}$, A. Lecavelier des Etangs ${ }^{3}$, J.-C. Bouret $^{1}$, A. Roberge ${ }^{4}$, \\ A. Vidal-Madjar ${ }^{3}$, C. Martin ${ }^{1}$, P. D. Feldman ${ }^{5}$, and R. Ferlet ${ }^{3}$ \\ ${ }^{1}$ Laboratoire d'Astrophysique de Marseille, BP 8, 13376 Marseille Cedex 12, France \\ 2 Université de Provence - CMI, 39 rue J. Curie, 13453 Marseille Cedex 13, France \\ 3 Institut d'Astrophysique de Paris, 98bis bd Arago, 75014 Paris, France \\ 4 Dept. of Terrestrial Magnetism, Carnegie Institution of Washington, 5241 Broad Branch Rd., \\ Washington, DC 20015-1305, USA \\ 5 Dept. of Physics and Astronomy, Johns Hopkins University, 3400 N. Charles St., Baltimore, \\ Maryland 21218, USA
}

Received 30 October 2003/ Accepted 27 December 2003

\begin{abstract}
We present the first far-UV spectra of the Herbig Be star HD 100546, observed by the Far Ultraviolet Spectroscopic Explorer (FUSE) as part of the Circumstellar Disks team program. We identified and analyzed numerous narrow absorption lines of circumstellar origin. Intense, broad and fairly asymmetric emission lines of C II, C III, O VI and S II were also detected, spanning temperatures up to $3 \times 10^{5} \mathrm{~K}$. Comparison of the spectra recorded two years apart reveals strong spectral variations, not only in the emission lines but also in the circumstellar lines of N I, N II, O I, Ar I and Fe II. The varying absorption lines of $\mathrm{N}$ I and O I exhibit the largest velocity width, from -200 to $+320 \mathrm{~km} \mathrm{~s}^{-1}$, relative to the star's rest-frame. Variations on a timescale of an hour occurred only in the N I resonance triplet and O I $\left({ }^{1} \mathrm{D}\right)$ lines. The spectroscopic variability highlighted by these two observations and the line profile analysis reveal signatures of both outflow and infall processes, which appear to be related in this system. We suggest that these spectral signatures originate in a stellar magnetosphere, which likely interacts with the innermost part of the star's circumstellar disk. This interpretation can account for the main features observed in the spectrum of HD 100546: variable emission and absorption lines as well as suspected continuum variation.
\end{abstract}

Key words. stars: circumstellar matter - stars: pre-main sequence - stars: individual: HD 100546 -

stars: emission-line, Be

\section{Introduction}

The present evolutionary scenario for protoplanetary disks around young stars predicts that they disperse in a few million years through planet formation and stellar activity. However in the early 1980s, IRAS observations demonstrated the presence of cold dust grains orbiting field A-type main sequence stars (Aumann et al. 1984). For one of them, $\beta$ Pictoris, direct imaging revealed that the circumstellar (CS) material is distributed in a large resolved disk (Smith \& Terrile 1984). The short dust lifetime compared to the star's age shows that the disk is composed of second generation material and could be generated by erosion and/or destruction of planetesimals orbiting the star (Vidal-Madjar et al. 1998).

Recent visible and near-IR coronagraphic observations give evidence for disk-like structures surrounding some old and isolated Herbig Ae/Be stars, which are suspected to be evolutionary precursors of $\mathrm{A}$ and $\mathrm{B}$ main sequence stars

Send offprint requests to: M. Deleuil, e-mail: magali.deleuil@oamp.fr
(Grady et al. 2000; Augereau et al. 2001). Such detections indicate that debris disks appear during the final stage of the pre-main sequence phase. However, this evolutionary scenario needs to be better constrained. In particular, the origin and the fate of the dust and gas, which must be decoupled in these late phases of planetary formation, need to be clarified. In addition, the structure of the CS environments - disk, envelope, or even a combination of both - around these pre-main sequence stars remains the subject of considerable controversy (e.g. Hillenbrand et al. 1992; Chiang et al. 2001; Pezzuto et al. 1997; Miroshnichenko et al. 1999).

HD 100546 is a nearby relatively isolated Herbig Be star. The presence of a CS disk was first suspected from its strong IR excess emission related to thermal emission from circumstellar dust grains (The et al. 1994). Recently, coronagraphic observations mapped an extended disk-like structure surrounding the star in the near-IR (Pantin et al. 2000; Augereau et al. 2001) and in the visible (Grady et al. 2001). The dusty component is characterized by numerous solid-state emission features in the IR spectral range (Waelkens et al. 1996), 
Table 1. Main astrophysical parameters. References: ${ }^{a}$ van den Ancker et al. (1998), ${ }^{b}$ Donati et al. (1997), ${ }^{c}$ Gerbaldi et al. (2001), ${ }^{d}$ Abt et al. (2002), ${ }^{e}$ Royer et al. (2002).

\begin{tabular}{llllllccc}
\hline \hline Star & $\begin{array}{l}\text { Spectral } \\
\text { type }\end{array}$ & $V$ mag & $\begin{array}{l}T_{\text {eff }} \\
(\mathrm{K})\end{array}$ & $E(B-V)$ & $\begin{array}{c}\text { Rad. vel } \\
\left(\mathrm{km} \mathrm{s}^{-1}\right)\end{array}$ & $\begin{array}{c}V \sin i \\
\left(\mathrm{~km} \mathrm{~s}^{-1}\right)\end{array}$ & $\begin{array}{c}\text { Age } \\
(\mathrm{Myr})\end{array}$ & $\begin{array}{c}\text { Distance } \\
(\mathrm{pc})\end{array}$ \\
\hline HD 100546, & B9Vne & 6.68 & 10500 & 0.080 & $+17 \pm 5$ & $65 \pm 5$ & $>10$ & 103 \\
HD 27638A $^{c, d}$ & B9 V & 5.39 & 10470 & 0.008 & 17.2 & 225 & 123 & 82 \\
HD 150100 $^{e}$ & B9.5 V & 5.53 & 10500 & 0.015 & -8.6 & 70 & - & 122.5 \\
\hline
\end{tabular}

Table 2. Observing log. The fourth column gives the number of subexposures. The $S / N$ ratios are given per resolution element.

\begin{tabular}{lccccc}
\hline \hline Star & Date & $\begin{array}{c}\text { Exp. time } \\
(\mathrm{s})\end{array}$ & $\#$ & $\begin{array}{c}S / N \\
1150 \AA\end{array}$ & $\begin{array}{c}S / N \\
1060 \AA\end{array}$ \\
\hline HD 100546 & $2000 / 03 / 26$ & 10347 & 2 & 43 & 17 \\
& $2002 / 03 / 03$ & 11940 & 3 & 49 & 17 \\
HD 27638 & $2001 / 01 / 02$ & 4347 & 9 & 91 & - \\
HD 150100 & $2002 / 07 / 13$ & 563 & 1 & 47 & - \\
\hline
\end{tabular}

with oxygen-rich and carbon-rich dust particles. Besides amorphous silicates, evidence of crystalline silicates and the striking similarity of its ISO spectrum to that of comet Hale-Bopp (Malfait et al. 1998) suggest that dust processing has occurred and strengthen the picture of an evolved system.

As a member of the Herbig Ae/Be group, the star shows Balmer and He I $5876 \AA$ lines in emission, with a high degree of variability on timescales from hours to days (Vieira et al. 1999). This spectral behavior has been interpreted as a sign of active mass exchange between the disk and the star. Transient redshifted absorption components have been also reported in the UV (Grady et al. 1996). Similarities between these sporadic accreting gas features and those routinely observed in $\beta$ Pictoris spectra led Grady et al. (1997) to attribute them to the evaporation of infalling cometary bodies. This interpretation has been however recently called into question on account of the strong radiation field and the likely stellar wind of the star (Beust et al. 2001).

With its rich CS environment and its apparent spectral analogies with $\beta$ Pictoris, HD 100546 is a prime target for our FUSE Circumstellar Disk team program (Moos et al. 2000). In a first paper (Lecavelier des Etangs et al. 2003), we focused on the disk molecular hydrogen content. Analysis of Lyman bands of this molecule in absorption reveals hot and dense gas, distributed in a thin layer close to the central star. In this paper, we complete the analysis of FUSE spectra to better characterize and constrain the CS environment of this star. The paper is organized as follows: Sect. 2 briefly describes the data acquisition and the reduction procedure for HD 100546 and two other stars that we used as references. Section 3 is devoted to absorption line identifications and analysis, after a brief description of the overall FUV spectra. Long and short-term variations in the absorption spectra are presented in Sect. 4. In Sect. 5, we analyze the emission line profiles. We discuss the nature and the possible origin of the spectral characteristics of the star and of the observed variability in Sect. 6. Conclusions are given in Sect. 7 .

\section{Observations and data reduction}

HD 100546 was observed twice by FUSE, in 2000 and in 2002. The observations were all made in the time-tagged mode, using the $30^{\prime \prime} \times 30^{\prime \prime}$ low-resolution aperture (LWRS). The individual spectra (905-1187 $\AA$ ) were processed with version 2.21 of the CALFUSE pipeline processing software (see Sahnow et al. 2000), which corrects for the main instrumental effects. As alignment of the channels may vary over time, the position of the star in the spectrograph entrance aperture may differ slightly from one exposure to the other, causing small shifts in the wavelength scale. To correct for this effect, the shifts between the individual sub-exposures were determined using a cross-correlation procedure and corrected before co-addition of channels. For HD 100546, the LiF $1 \mathrm{~b}$ spectrum was affected by the so-called "worm artifact" in the detector, which artificially decreases the flux level in this segment. This phenomenon was present in both exposures and at different wavelength locations. The LiF $1 b$ spectrum was thus used only to confirm the presence of lines observed in the $\mathrm{LiF} 2 \mathrm{a}$ channel but not for the spectral line analysis. The co-added spectra in each channel were rebinned, to increase the $S / N$ ratio without degrading the resolution, estimated to be about $17 \mathrm{~km} \mathrm{~s}^{-1}$ in the $\mathrm{LiF}$ channel $(R=\lambda / \Delta \lambda=18000)$. An overview of the whole FUSE spectrum is shown in Fig. 1.

The absolute wavelength scales were set by comparing the long wavelength parts of the $\mathrm{LiF} 2 \mathrm{a}$ segment spectra with a rotationally broadened Kurucz stellar model with the appropriate stellar parameters (Table 1) and assuming a solar metallicity. The LiF 2a segment (1090-1180 $\AA$ ) is the most suitable for that task, with prominent and marked photospheric lines. We found that both HD 100546 spectra were well calibrated in the star's reference frame, with an uncertainty of $\pm 5 \mathrm{~km} \mathrm{~s}^{-1}$.

For the other segments, the offset was established using the very numerous narrow $\mathrm{H}_{2}$ molecular lines observed in all segments (Lecavelier des Etangs et al. 2003). For the data analysis presented in the following sections, we used the individual channels independently and not the co-added spectra.

To disentangle the CS spectral features from photospheric ones and to assess the continuum level of HD 100546, we also used HD 27638 and HD 150100 as reference stars. The CS and IS absorption lines are easy to identify, as they are much narrower than photospheric lines. The use of a reference spectrum was however particularly useful for the strong $\mathrm{C}$ I blends and the N I triplet lines analysis (see Sect. 3). 


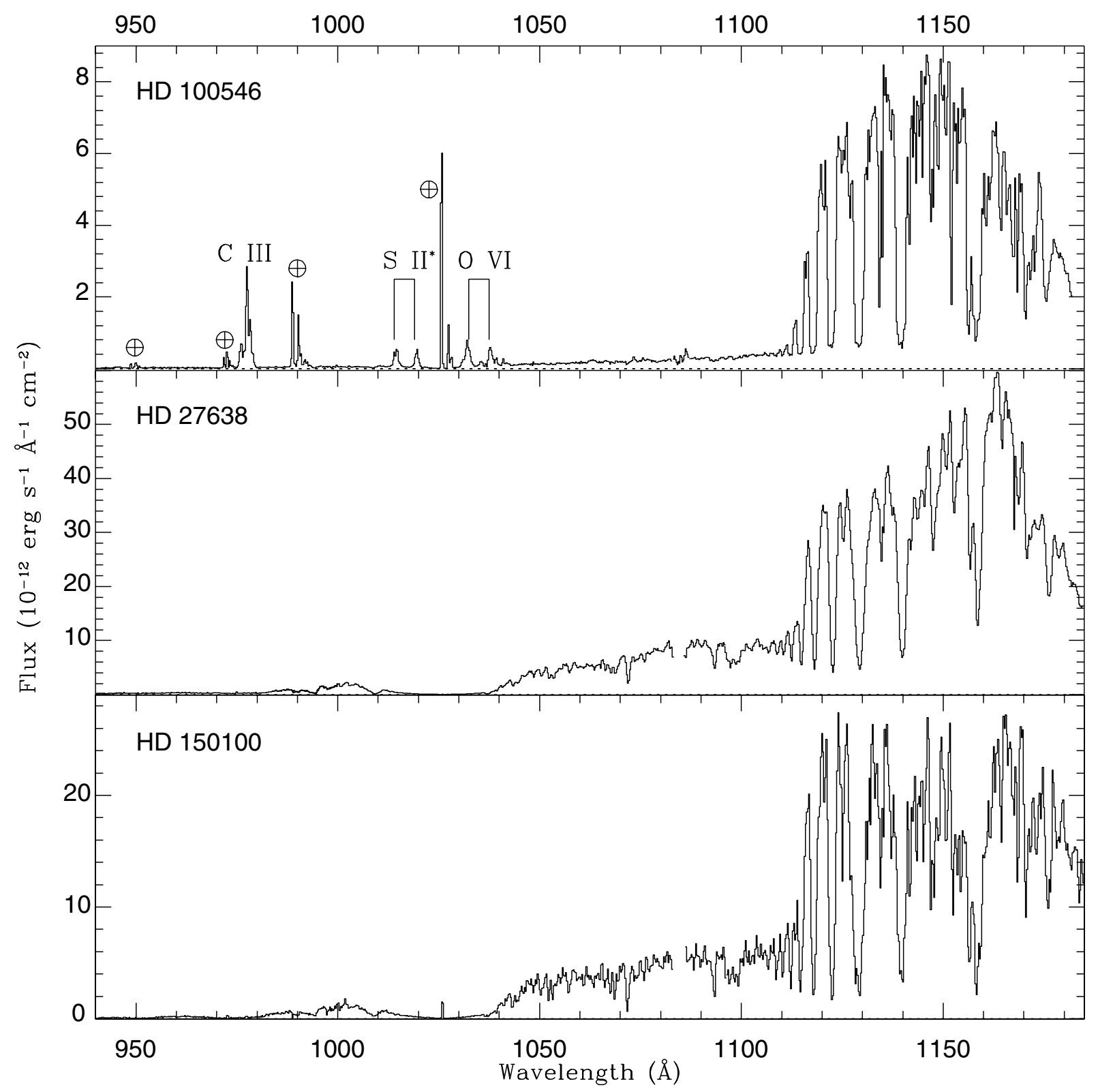

Fig. 1. The FUSE spectra of HD 100546 (recorded in 2002) and the two other B-stars used as a reference for the photospheric spectrum analysis. For clarity, the spectra plotted in this figure have been rebinned by 40 pixels. In the HD 100546 spectrum, the main emission features are indicated and the brightest airglow lines are labelled with the $\oplus$ symbol. The prominent absorption features between 1110 and $1160 \AA$ are photospheric C I lines.

HD 27638 is a B9 main sequence star, observed in the context of our GTO FUSE program. This star is a member of a binary system, consisting of a post $\mathrm{T}$ Tauri secondary and a B-type primary, with a separation of 19.4" (Gerbaldi et al. 2001). The measured projected rotation velocity of the primary is $225 \mathrm{~km} \mathrm{~s}^{-1}$, close to the value for HD 100546 quoted by Grady et al. (1996).

The HD 150100 FUV spectrum was retrieved from the FUSE archives. It is a B9.5 main sequence star in a visual triple system separated by about 90 arcsec, with a white dwarf companion (Barstow et al. 2002). This star also exhibits a few similarities with HD 100546 in terms of effective temperature, reddening and $v \sin i=70 \mathrm{~km} \mathrm{~s}^{-1}$, (Royer et al. 2002) similar to the value derived by Donati et al. (1997) for HD 100546 (see
Table 1). A brief summary of the relevant stellar parameters is given in Table 1 and the log of observations in Table 2.

\section{Overview of the FUV spectrum and $C S$ absorption line analysis}

The HD 100546 FUSE spectrum is characterized by a very low flux level at short wavelengths, with strong and broad emission lines due to ionized species (Fig. 1). As expected from its effective temperature, in the long wavelength range the photospheric spectrum is well-developed and dominated by broad C I absorption lines between $1110 \AA$ and $1160 \AA$. Above $1150 \AA$, the extended blue wing of the Ly $\alpha$ absorption line dims the continuum. Below $1120 \AA$, the continuum drops sharply to a low flux 
Table 3. Derived parameters for the CS species identified in absorption in HD 100546 spectrum. The velocities are given in the star's rest frame. "sat" is for saturated lines, "var" for variability. For the saturated absorption lines, we measured the velocity of the centroid. For the species which present no temporal spectral variation, we give the velocity and the column density calculated from the average of the two spectra.

\begin{tabular}{|c|c|c|c|c|}
\hline \multirow[t]{2}{*}{ Element } & \multirow{2}{*}{$\begin{array}{l}\text { Level } \\
\left(\mathrm{cm}^{-1}\right)\end{array}$} & \multicolumn{2}{|c|}{ Velocity $\left(\mathrm{km} \mathrm{s}^{-1}\right)$} & \multirow{2}{*}{$\begin{array}{c}\log N \\
\left(\mathrm{~cm}^{-2}\right)\end{array}$} \\
\hline & & 2000 & 2002 & \\
\hline $\mathrm{C}_{\mathrm{II}}$ & 0.00 & $-12 \pm 4$ & $-11 \pm 4$ & var/sat \\
\hline $\mathrm{C} \mathrm{II}^{*}$ & 63.42 & $-12 \pm 4$ & $-11 \pm 4$ & $\mathrm{var} / \mathrm{sat}$ \\
\hline C III & 0.00 & $-11 \pm 5$ & $-19 \pm 5$ & var/sat \\
\hline N I & 0.00 & \multicolumn{2}{|c|}{$6.8_{-0.6}^{+0.7}$} & $17.73_{-0.13}^{+0.19}$ \\
\hline $\mathrm{NI}^{2} D_{5 / 2}$ & 19224.46 & \multicolumn{2}{|c|}{ “ } & var \\
\hline $\mathrm{N} \mathrm{I}^{2} D_{3 / 2}$ & 19233.18 & \multicolumn{2}{|c|}{ “ } & var \\
\hline $\mathrm{NI}^{2} \mathrm{P}$ & 28839. & \multicolumn{2}{|c|}{ “ } & var \\
\hline $\mathrm{N}_{\text {II }}$ & 0.00 & $-5 \pm 3$ & $-11.6 \pm 2$ & var/sat \\
\hline $\mathrm{N}_{\text {II }}^{*}$ & 48.67 & $"$ & $"$ & var/sat \\
\hline $\mathrm{N}$ II $^{* *}$ & 130.80 & $"$ & $"$ & $\mathrm{var} / \mathrm{sat}$ \\
\hline OI & 0 & $+3 \pm 5$ & $-11 \pm 5$ & var/sat \\
\hline $\mathrm{OI}^{*}$ & 158.26 & $+3 \pm 5$ & $-11 \pm 5$ & $\mathrm{var} / \mathrm{sat}$ \\
\hline $\mathrm{OI}^{* *}$ & 226.97 & $3 \pm 5$ & $-11 \pm 5$ & var/sat \\
\hline $\mathrm{O} \mathrm{I}^{1} \mathrm{D}$ & 15867.86 & & & var/sat \\
\hline P II & 0.00 & \multicolumn{2}{|c|}{$7.6_{-1.7}^{+1.1}$} & 14.43 \\
\hline P II ${ }^{*}$ & 164.90 & \multicolumn{2}{|c|}{$"$} & 13.17 \\
\hline $\mathrm{P}_{\mathrm{II}^{* *}}$ & 469.12 & \multicolumn{2}{|c|}{$"$} & 12.87 \\
\hline ArI & 0.00 & $-7 \pm 4$ & $-12 \pm 2$ & var/sat \\
\hline Mn II & 0.00 & \multicolumn{2}{|c|}{$9.80_{-1.4}^{+1.5}$} & $14.10_{-0.07}^{+0.07}$ \\
\hline Fe II & 0.00 & \multicolumn{2}{|c|}{$7.8_{-0.02}^{+0.01}$} & $15.58_{-0.05}^{+0.16}$ \\
\hline $\mathrm{Fe} \mathrm{II}^{*}$ & 384.79 & \multicolumn{2}{|c|}{$"}$, & $14.58_{-0.33}^{+0.52}$ \\
\hline Fe II** & 667.68 & \multicolumn{2}{|c|}{$"$} & $13.95_{-0.01}^{+0.01}$ \\
\hline $\mathrm{Fe} \mathrm{II}^{* * *}$ & 862.61 & \multicolumn{2}{|c|}{$"$} & $13.38_{-0.01}^{+0.01}$ \\
\hline $\mathrm{Fe} \mathrm{II}^{* * *}$ & 977.05 & \multicolumn{2}{|c|}{$"$} & $13.95_{-0.1}^{+0.1}$ \\
\hline
\end{tabular}

level. This sudden flux decrease between $1110 \AA$ and $1130 \AA$, which is not predicted by classical LTE spectral models, will be discussed in Sect. 3.3. Due to the controversial $v$ sin $i$ estimates, we compared the HD 100546 FUSE spectra to those of the reference stars. We argue that a high $v \sin i$ value is excluded because small absorption lines in the photospheric spectrum are smoothed and vanish in that case.

Numerous narrow absorption lines are superimposed on the rotationally broadened photospheric spectrum and on the emission lines (Sect. 5). Most of them are due to electronic transitions of $\mathrm{H}_{2}$ (Lecavelier des Etangs et al. 2003). The other lines correspond to transitions of atomic and ionic species arising not only from resonance levels but also from fine-structure and even metastable levels. For resonance lines, we used wavelengths and oscillator strengths tabulated by Morton (2000); otherwise, we used the Atomic Line List kindly provided by P. van Hoof

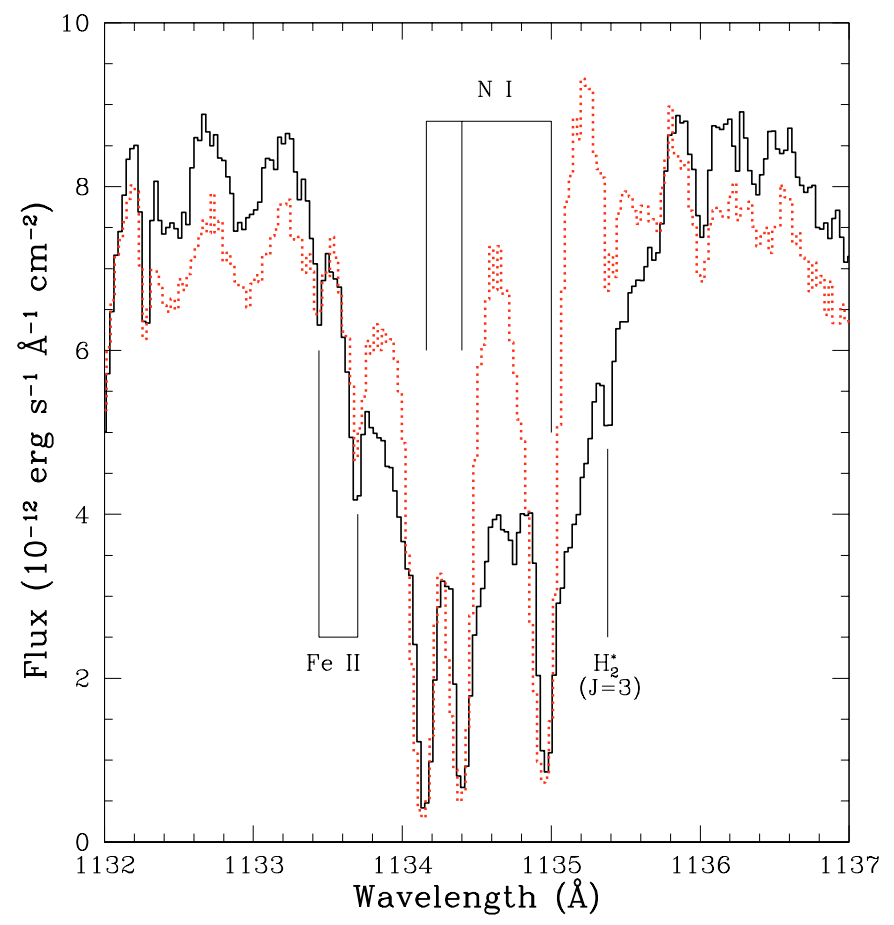

Fig. 2. Comparison of HD 100546 spectra recorded two years apart (2000 - solid line; 2002 - dotted line), in the spectral range of the $\mathrm{N}$ I triplet lines. The neighboring CS lines are labeled. Note the variations in the flux level, while the expected stellar continuum level in 2000 is close to the 2002 level. This indicates that extra-absorption occurred in the 2000 spectrum, longward and shortward of the stellar rest velocity.

(URL: http://www.pa.uky.edu/ peter/atomic/). We identified atomic lines from volatiles (carbon, nitrogen and oxygen) and a number of mid-refractory and refractory elements (Ar I, Mn II, P II and Fe II) (see Table 7). The CS origin of the observed lines was established by (i) the presence of lines arising from excited levels, and (ii) the agreement with the star's radial velocity, within the uncertainty of the FUSE absolute wavelength calibration. Furthermore, despite the premain sequence status of the star, its reddening is low and the line of sight appears devoid of dense and hot interstellar clouds. Indeed, the star is probably in front of an interstellar cloud and most of the estimated reddening is due to the circumstellar matter (Malfait et al. 1998; Vieira et al. 1999).

For a given element, when possible, we estimated the column densities and the radial velocities using all unsaturated lines simultaneously, while the line widths, $b$, were measured from saturated lines. To do this, we used the "Owens" package developed by Lemoine (Lemoine et al. 2002), who kindly provided it to us. The derived values are listed in Table 3 and our results are summarized below.

\subsection{Nitrogen}

Nitrogen lines from both neutral and the first ionization state are detected. Not only the well-developed N I resonance triplet at $1134 \AA$ is observed (Fig. 2), but also much weaker lines from the ground level at 1159 and $1160 \AA$ (UV multiplet 1.01). 
In addition, absorption lines arising from the metastable levels $\mathrm{N} \mathrm{I}^{2} \mathrm{D}$ at $2.38 \mathrm{eV}$ and ${ }^{2} \mathrm{P}$ at $3.58 \mathrm{eV}$ are also present. All nitrogen lines, except the weaker UV1.01 resonance lines, show significant variations (see Sect. 4). Therefore, only the two weak UV1.01 lines were used to derive the column density and the velocity of the gas.

$\mathrm{N}$ II resonance lines, arising from excited-J levels, are also detected in absorption in both $\mathrm{SiC}$ segments, making the detection reliable despite the low sensitivity at the ends of these segments. The FUSE resolution is however not sufficient to observe the exact line-profile, as the multiplet is a blend of 5 components. The lines appear broad and blueshifted relative to the star's rest frame. In addition, time variations in the line profiles are suspected (see Sect. 4.1.2).

\subsection{Oxygen}

O I fine-structure lines are seen in absorption, superimposed on the C III emission line at $977 \AA$ (see Sect. 5). The detection of other resonance transitions in the $\mathrm{LiF} 1 \mathrm{a} / 2 \mathrm{~b}$ spectra is difficult due to the low photospheric flux level and airglow emission. However, the O I $\left({ }^{1} \mathrm{D}\right)$ metastable transition at $1152.15 \AA$ is clearly present (Fig. 7). All these lines are broad and, like the neutral nitrogen lines, are affected by temporal variations.

\subsection{Carbon}

The presence of neutral carbon lines from circumstellar gas is suspected, following previous detection by Grady et al. (1997). The FUSE spectral resolution is insufficient to resolve the multiple blends at the bottom of strong photospheric lines. However, two points favor the presence of CS C I: (i) the shape of the C I blends; (ii) the break in the spectral energy distribution (SED) at $1110 \AA$.

In the HD 100546 spectra, the carbon blends are surprisingly strong with nearly flattened bottoms. As illustrated in Fig. 3, none of the reference spectra exhibit such flat-bottomed absorption features, nor does $51 \mathrm{Oph}$, another target of our CS Disk program with similar $T_{\text {eff }}$ (see Roberge et al. 2001). As the $v \sin i$ estimates for HD 100546 ranged from $65 \mathrm{~km} \mathrm{~s}^{-1}$ (Donati et al. 1997) to $250 \mathrm{~km} \mathrm{~s}^{-1}$ (Grady et al. 1996), we checked that such wide and flattened $C_{I}$ blends could not be explained by stellar rotation. In fact, a large increase in the $v \sin i$ value leads to small changes in the C I blends shapes but to a rather noticeable decrease in the mean photospheric level (Fig. 3).

In addition, as Fig. 3 shows, a sharp break occurs in the SED of HD 100546 around $1115 \AA$. The position of this break is close to the C I ionization edge at $1102.7 \AA$ and we thus suggest that this abrupt drop in the continuum of the star is due to absorption by $\mathrm{CI}$ in the ground state. We examined stars in the FUSE archives with temperatures in the range 10000 to $11500 \mathrm{~K}$. We found that all those with $T_{\text {eff }}$ around $10500 \mathrm{~K}$ present this sharp decrement. But in the case of HD 100546, this $C$ I break appears more prominent than in other stars and leads to an unusual low flux level below $1115 \AA$.

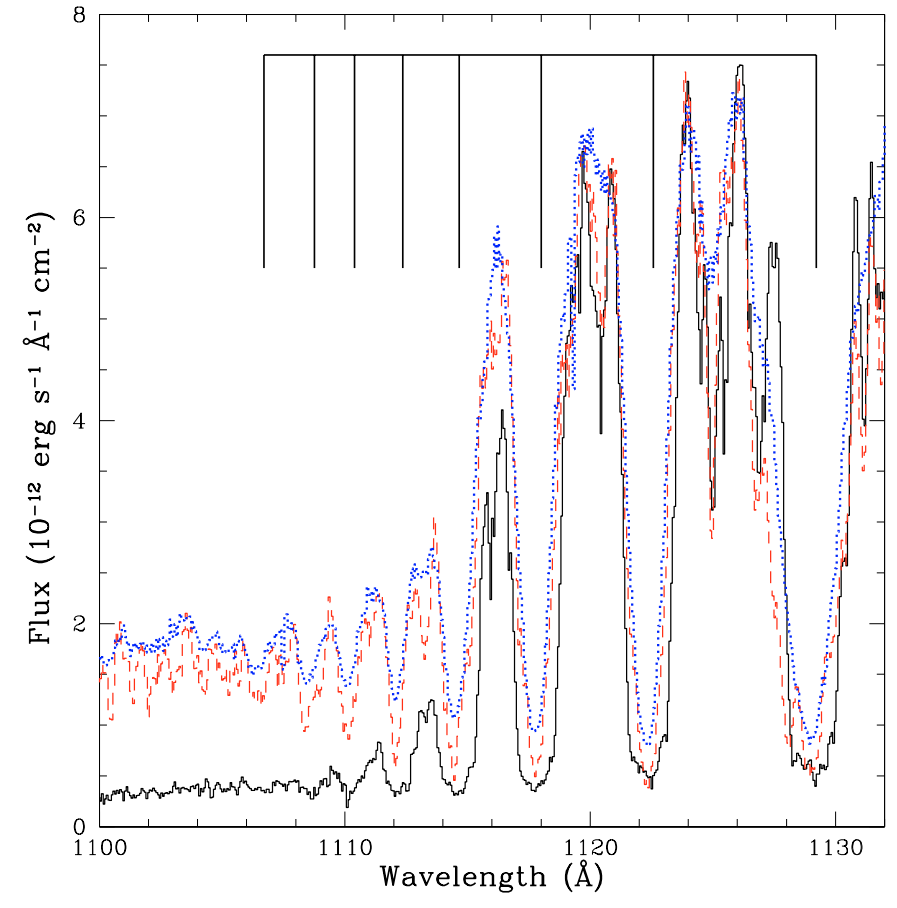

Fig. 3. The 1100 to $1132 \AA$ spectral range for HD 100546 observed in 2000 (solid line), HD 27638 (dotted line) and HD 150100 (dashed line). The flux of the two reference stars has been scaled to HD 100546 at about $1120 \AA$ and co-aligned. All three spectra have been rebinned by 10 pixels for this plot. The main $\mathrm{C}$ I blends are labeled at the top.

These qualitative results are strengthened by the fact that the HD 100546 FUSE spectrum does not agree with either LTE or NLTE stellar models, unless the carbon abundance is very high in the star, which is difficult to account for at the present stage of the analysis.

These arguments provide clues to the presence of a carbonrich CS environment. It would result in unresolved CS C I absorption lines and an appreciable contribution to the continuous absorption. However, the detailed analysis of the line profiles deserves further modeling of a complete set of stellar spectra, sampling a wide range in temperature (Bouret et al., in preparation).

In addition, the $\mathrm{C}$ II resonance doublet appears in absorption superimposed on a low and highly uncertain flux level provided by a blend of at least two emission lines at $1035 \AA$ (Sect. 5). These absorption lines are broad; even if an interstellar (IS) contribution can not be excluded, these lines are related to the star's CS environment.

\subsection{Argon}

Despite the low signal to noise ratio in the corresponding spectral range due to the low continuum level, the two Ar I resonance doublet transitions are clearly observed in the four segments. They appear as broad absorption lines clearly blueshifted relative to the other CS lines in both spectra. In addition, strong variations are detected between 2000 and 2002, excluding a purely interstellar origin (Fig. 4). 


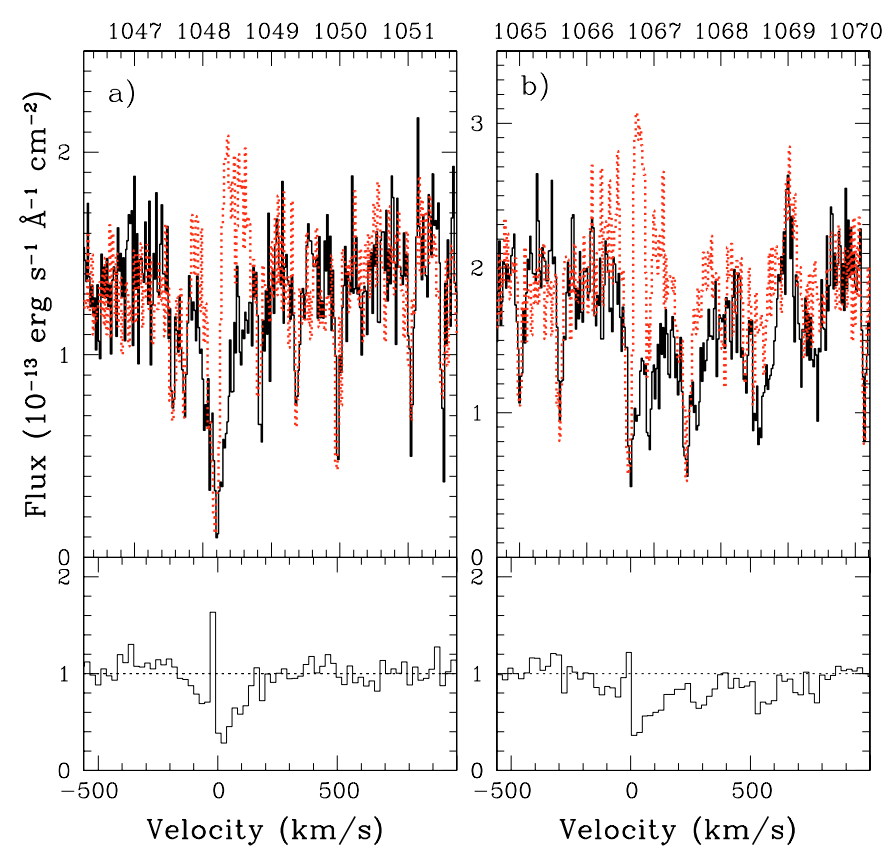

Fig. 4. Spectral variations in ArI resonance doublet lines at a) $1066.6 \AA$ and b) $1048.2 \AA$. The top panels show the data recorded in 2000 (solid line) and in 2002 (dotted line). The 2000 spectrum divided by the 2002 one is shown in the bottom panels. The velocity scale is in the rest frame of the star.

\subsection{Phosphorus}

P II resonance lines, as well as fine structure lines from energy levels up to $469 \mathrm{~cm}^{-1}$, are detected in HD 100546 LiF $2 \mathrm{a} / 1 \mathrm{~b}$ spectra (see Fig. 7). These lines are not saturated and show no variation between the spectra recorded two years apart. In this case, the column densities for each level were determined from the average of the two spectra to increase the $S / N$ ratio, but we cannot derive the intrinsic line width.

\subsection{Manganese}

Three resonance lines of Mn II are observed in absorption against the well-developed photospheric spectrum in the long wavelength portion of the FUSE wavelength range. As for P II, the lines are weak and appear unsaturated with no timevariation and the column densities were measured from the averaged spectrum. In both spectra, the $1164.208 \AA$ line appears with a different velocity than the two other resonance lines. The same phenomenon was observed in the FUSE spectra of two other stars, HD 259431 and HD 250550 (Bouret et al. 2003). This is likely due to an error in the theoretical wavelength of this transition.

\subsection{Iron}

Several Fe II absorption lines arising from resonance fine structure levels up to $977 \mathrm{~cm}^{-1}$ are detected. The large number of lines arising from the same energy level but differing in oscillator strength permitted an accurate column density determination. However, comparison of the two spectra with each other and with our reference spectrum shows that the line profiles of the strongest lines are not symmetric, but exhibit additional extended absorption features (Fig. 8). This is conspicuous for the strongest line at $1144.9 \AA$ (Sect. 4.1.2). As we used the weakest unsaturated lines to determine the column densities, they are fairly well determined even for the ground level. For this ion, the few saturated lines arising from the fine structure levels were used to measure the intrinsic line width. We found $b=1.1_{-0.1}^{+0.7}$, where the error bars are $2-\sigma$ error bars, calculated from the classical $\chi^{2}$ statistic.

Concerning Fe III, the strong C I blend at $1122 \AA$ prevents us from deriving any firm conclusions concerning the presence of this ion. At the flat bottom of this blend, the Fe III resonance line may be marginally detected in the 2000 spectrum but does not clearly appear in the 2002 one.

As a first conclusion, our analysis of the HD 100546 spectra shows that neutral and first ionization species are the dominant phase of the circumstellar gas. Furthermore, several absorption lines arising from metastable species of volatile elements were seen: NI $\left({ }^{2} \mathrm{D}\right), \mathrm{N}$ I $\left({ }^{2} \mathrm{P}\right)$ and O I $\left({ }^{1} \mathrm{D}\right)$. The presence of these lines confirms the CS nature of the observed gas. As noted by Roberge et al. (2001), the lifetimes of these metastable levels are about a few hours, which indicates that they should be continuously re-populated. These lines should thus originate in a warm, collisionally heated gas.

\section{Temporal variations}

Comparison of the two FUSE spectra of HD 100546 reveals variations not only in circumstellar absorption lines as previously reported by Grady et al. (1997) in the IUE UV spectral range, but also in the photospheric flux itself and in the emission lines. For the latter, their temporal behavior will be discussed in detail in Sect. 5, together with their profile analysis. In this section, we will focus on continuum and absorption line variations.

\subsection{Long-term variability}

\subsubsection{Continuum}

The direct comparison of the spectra taken two years apart in the two independent LiF channels ( $1 \mathrm{~b}$ and $2 \mathrm{a}$ ) where the $S / N$ is the highest, shows significant variations in the continuum flux level. Between $1100 \AA$ and $1180 \AA$, the photospheric flux level can vary by more than $10 \%$, a value in agreement with the photometric variations observed by Hipparcos $\left(\delta H_{\mathrm{p}} \simeq 0.19^{\mathrm{m}}\right.$, van den Ancker et al. 1998). The reality of these variations is however difficult to assess, as it is not possible to find a large spectral range of continuum devoid of strong photospheric lines. In spite of the long time interval between the two observations, it cannot be due to a change in the instrument performance. All spectra were processed with the same version of the FUSE pipeline, which takes into account the time evolution of the instrument sensitivity. These variations need however to be further confirmed by a time series of observations.

Such photometric variations are commonly seen in HAeBes stars. As with polarimetric variability, their exact origin in young stars is still under debate. If polarization 
variability (Clarke et al. 1999) originates in gaseous and/or dusty circumstellar shells as discussed by Yudin (2000), photometric and spectral variations with winds, chromospheric signatures or even discrete accretion features, are probably driven by the star itself (Catala et al. 1993; Boehm \& Catala 1995).

\subsubsection{CS absorption lines}

Long-term variations in CS absorption lines between the 2000 and 2002 data are seen. Nearly all volatile spectral lines we observed, including lines from the $\mathrm{NI}^{2}\left({ }^{2} \mathrm{D}\right)$ and $\left({ }^{2} \mathrm{P}\right)$ levels and $\mathrm{OI} \lambda 1152$, exhibit noticeable temporal variations. These levels correspond to excitation temperatures of about 28000 , 41000 and $23000 \mathrm{~K}$ respectively. For the heavier elements, only the strongest transition of Fe II and the Ar I resonance lines show clear evidence of variations.

The spectroscopic variability in the N I lines occurs over a large velocity range. To estimate the stellar continuum level, HD 100546 spectra were compared to the reference spectra and to a synthetic spectrum calculated with the star's photospheric parameters. This comparison shows that the continuum around the nitrogen triplet is high and close to the apparent continuum flux level in the 2002 spectrum. Using the MDRS slit off-target exposures, we checked that this variation is not due to the N I airglow contamination, which is much lower that the amplitude of the variation.

The CS N I absorption feature is composed of at least two components: a main saturated central component, and a very broad component longward and shortward of the star's rest frame, with no resolved features (Fig. 2). The central absorption, observed nearly at the star's radial velocity, could be a mixture of unresolved IS and CS absorption features. In addition, the 2002 spectrum presents excess absorption shortward of the main absorption feature, which results in a blueshifted emission feature in the division (Fig. 5). The velocity extent of the broad component has been estimated using the red wing of the $\lambda 1134.98$ triplet, and the blue wing of the $\lambda 1134.16$ line (see Table 4). Considering the wide velocity range of the variations, and the fact that the spectral resolution does not allow us to resolve the structure, we did not perform any abundance analysis of the variable gas. Moving to higher excitation levels, the varying features are less developed in velocity extent and strength.

Despite very low $S / N$ ratios at the ends of the $\mathrm{SiC} 1 \mathrm{a}$ and $2 \mathrm{~b}$ spectra, the comparison of the two epoch spectra shows the presence of additional extended redshifted emission in 2002 in the resonance N II multiplet (Fig. 6). This feature is observed in both $\mathrm{SiC}$ segments, which makes its detection reliable. The centroids of the absorption features, estimated from the bluest line of the multiplet, are blueshifted (see Table 3). This spectral behavior is similar to that observed in the Ar I resonance lines (Fig. 4). Both ions appear related to gas moving outwards from the star.

Concerning the $\mathrm{OI}\left({ }^{1} \mathrm{D}\right)$ transition, comparison of the line profiles revealed dramatic spectral variations (see Fig. 7). Extra circumstellar absorption occurred longward of the stellar rest wavelength in the 2000 spectrum, and shortward in the

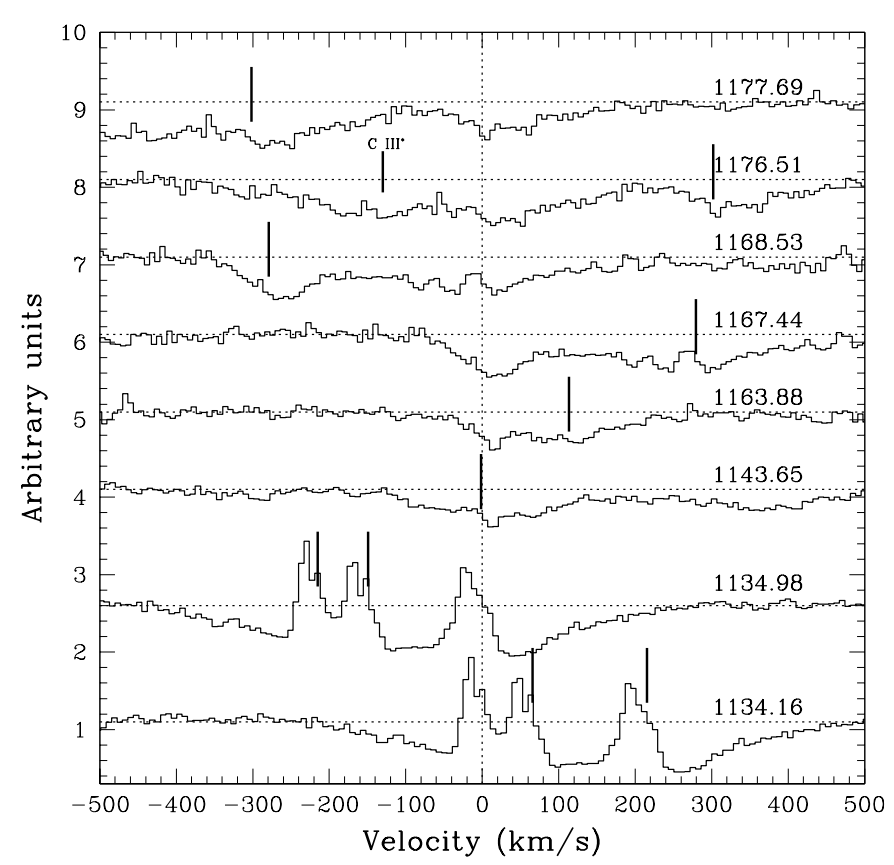

Fig. 5. Long-term variations of N I observed in the HD 100546 FUSE spectra. The division of the 2000 spectrum by the 2002 one in the LiF $2 \mathrm{a}$ channel, rebinned by 4 pixels, is plotted in the spectral range of the various $\mathrm{N}$ I lines on velocity scale. The full vertical lines indicate the position of other nearby $\mathrm{N}$ I lines.

Table 4. Long-term variable CS spectral features in HD 100546 spectrum. $\beta$ is the ratio of the radiation pressure to the stellar gravity. For Fe II, the $\beta$ value listed is taken from Beust et al. (2001).

\begin{tabular}{lccc}
\hline \hline Ion & $\begin{array}{c}\text { Energy level } \\
\left(\mathrm{cm}^{-1}\right)\end{array}$ & $\begin{array}{c}\text { Vel. range } \\
\left(\mathrm{km} \mathrm{s}^{-1}\right)\end{array}$ & $\beta$ \\
\hline N I & 0.00 & $-200 ;+300$ & $10.1 \pm 0.3$ \\
N I $\left({ }^{2} \mathrm{P}\right)$ & 28839 & $? ;+150$ & $\ldots$ \\
N I $\left({ }^{2} \mathrm{D}\right)$ & 19228 & $-60 ;+150$ & $\ldots$ \\
N II 1084 & $0.0,48.7,130.8$ & $-60 ;+240$ & $0.51 \pm 0.05$ \\
O I $\left({ }^{1} \mathrm{D}\right)$ & 15867.8 & $-180 ;+320$ & $4.263 \pm 0.075$ \\
Ar I & 0.00 & $-170 ;+180$ & $0.083 \pm 0.008$ \\
Fe II 1144.93 & 0.00 & $-40 ;+110$ & $46.6^{a}$ \\
\hline
\end{tabular}

2002 spectrum. Again, the line seems to be composed of at least two spectral components: a narrow feature at the star's velocity and a broad extended one. We also observed variations in the O I fine structure lines seen in absorption against the $\mathrm{C}$ III emission line at $977 \AA$. But in this spectral range, the variations of the emission line profile prevent their analysis.

The strongest Fe II resonance line at $1144.9 \AA$ appeared to vary significantly between the two years of observation (Fig. 8). For this line, the 2000 spectrum shows excess absorption which extends over more than $100 \mathrm{~km} \mathrm{~s}^{-1}$ longward of the star's velocity. The other Fe II resonance lines do not show clear variations. They are weaker by a factor of more than 5.6, and in addition, the strongest line is blended with N I lines. This indicates that the varying Fe II gas is unsaturated. 


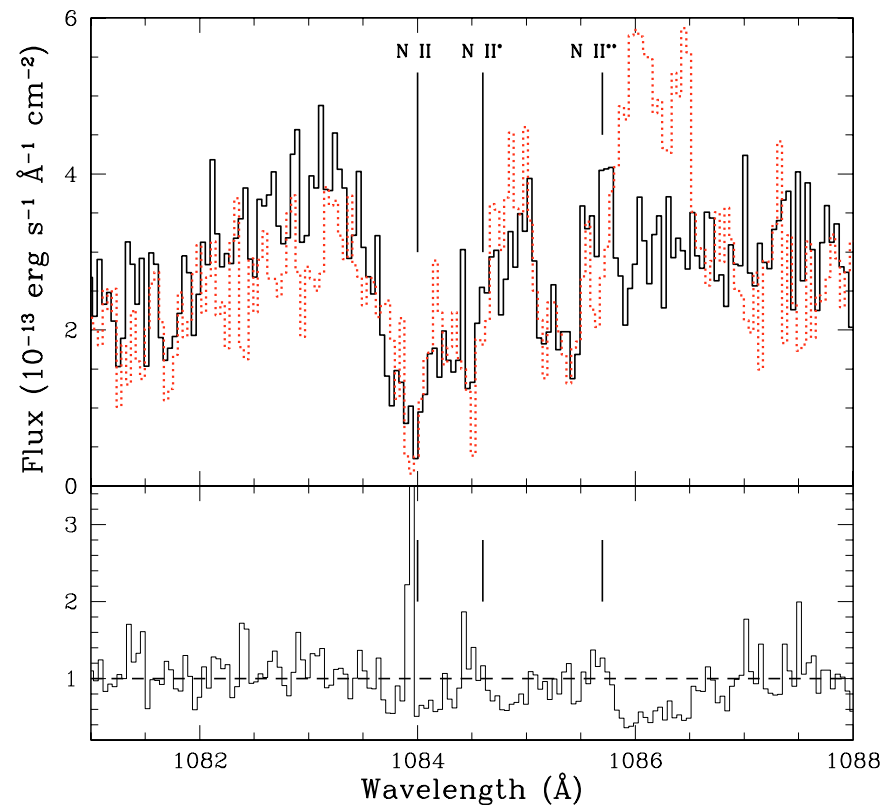

Fig. 6. Long-timescale variations of N II in the HD 100546 FUSE spectra. The spectra from the $\mathrm{SiC} 1$ a channel, rebinned by 7 pixels, observed in 2000 (solid line) and in 2002 (dotted line) are shown at the top. The 2000 spectrum divided by the 2002 one is shown at the bottom. The position of the strongest lines of the multiplet in the stellar rest frame are indicated.

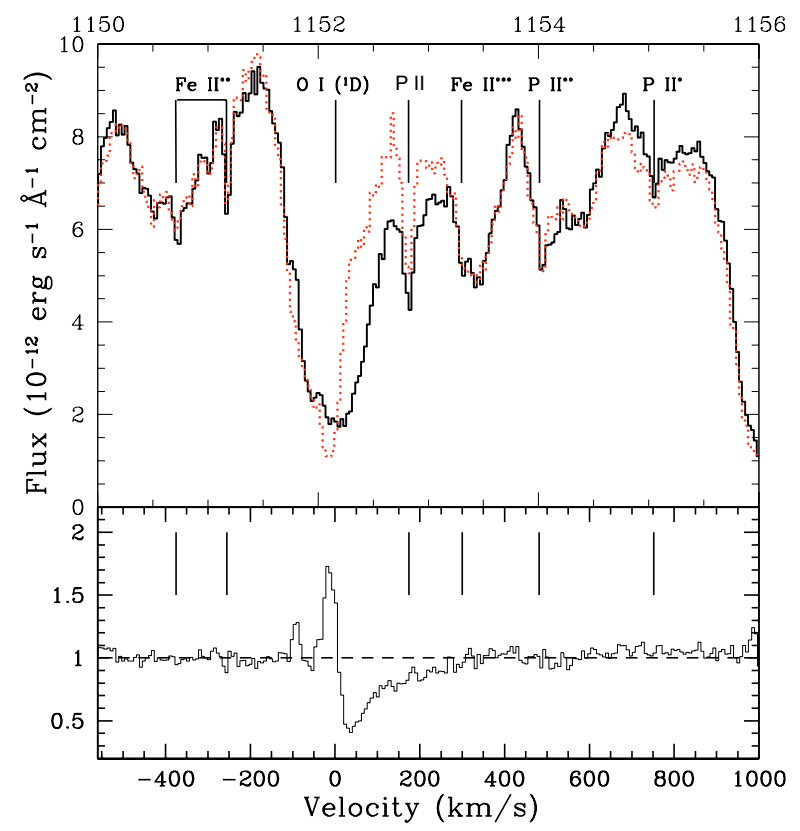

Fig. 7. Spectral variations in the OI $\left({ }^{1} \mathrm{D}\right)$ line at $1152.15 \AA$ in the LiF 2a segment (solid line: 2000 spectrum; dotted line: 2002 spectrum). Nearby circumstellar absorption lines are indicated.

\subsection{Short-term variations}

The $S / N$ ratio of the individual sub-exposures is high enough to search for short-term variations. For each year of observation, we divided the first exposure by the second, and also by the third one in the case of the 2002 visit where three subexposures were taken.
We detect no short-term variations during the 2000 observations: the two exposures recorded one hour and a half apart are the same within the noise level. On the contrary, the first 2002 sub-exposure divided by the two other ones successively clearly shows spectral variations in the strong N I triplet and O I ${ }^{1} \mathrm{D}$ absorption lines (Fig. 9). No other short-term spectral variation is detected in the FUV spectral domain.

In N I lines, the short-term variation corresponds to an extra absorption in the first sub-exposure relative to the other two. The bulk of this extra absorption is close to the star's radial velocity (Table 5) or slightly blueshifted. Its depth decreases in time, on a timescale of about one hour. Furthermore, the equivalent widths of the individual variable features are not proportional to the corresponding oscillator strengths, indicating that the variable gas should be saturated. In addition, in both cases the varying features exhibit nearly the same depth without reaching the instrumental scattered light level, indicating that the absorbing gas does not cover the full stellar disc. The covering factor is about $20 \%$ in the first division and increases to $50 \%$ in the second one.

The spectral variations of the O I $\left({ }^{1} \mathrm{D}\right)$ line occurred mainly between the first and the second exposures, with extra absorption in the second sub-exposure, slightly blueshifted relative to the star's velocity. This feature disappears in the third exposure. The present data allow us to draw a few conclusions:

- The short-term variable gas is warm, with an excitation temperature around $20000 \mathrm{~K}$ as evidenced by the presence of the O I $\left({ }^{1} \mathrm{D}\right)$ line. In any case, the temperature is greater than $28000 \mathrm{~K}$, as no short-timescale variation is observed in lines arising from $\mathrm{N}_{\mathrm{I}}\left({ }^{2} \mathrm{D}\right)$.

- For OI and NI, the data show excess absorption in a velocity range blueshifted with respect to the star's velocity, with no noticeable change in radial velocity between the sub-exposures, at the FUSE resolution accuracy.

- The variable NI lines are saturated, which shows that the gas is related to an optically thick medium. At the time of our observations, the covering factor is decreasing in time.

This indicates that an optically thick, clumpy outflow of gas at low velocity, whose size was decreasing in time, occurred in 2002 .

\section{Emission lines}

\subsection{Emission lines: Overview}

One of the most striking characteristics of the FUV spectra of this star is the presence of emission lines superimposed on the low continuum level (Fig. 10). These lines correspond to resonance or excited transitions of moderate and highly ionized species which cannot be created by simple stellar photoionization. O VI and C III resonance lines are the most prominent emission features in the spectra.

Emission lines in transitions from excited levels are also observed in the two strongest $\mathrm{S}$ II lines in the FUSE spectral domain, at 1014.44 $\AA$ and $1019.53 \AA$. These lines arise from the metastable $\left({ }^{2} \mathrm{D}\right)$ level. We cannot exclude the presence of the C III* multiplet at $1176 \AA$, although the intense photospheric 


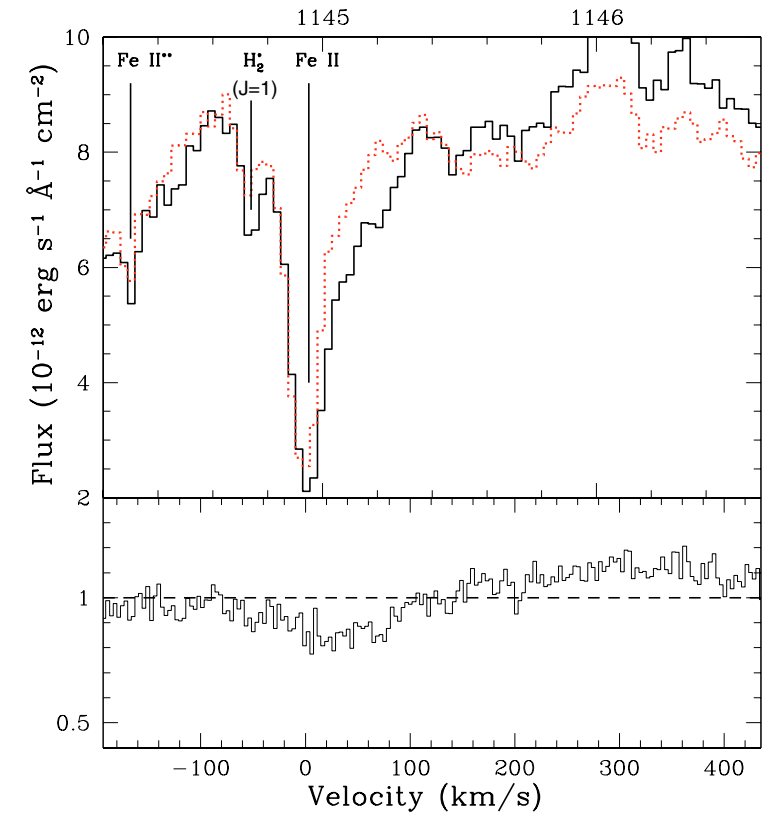

Fig. 8. Same data as in Fig. 7 but in the region around the strongest Fe II line at $1144.9 \AA$.

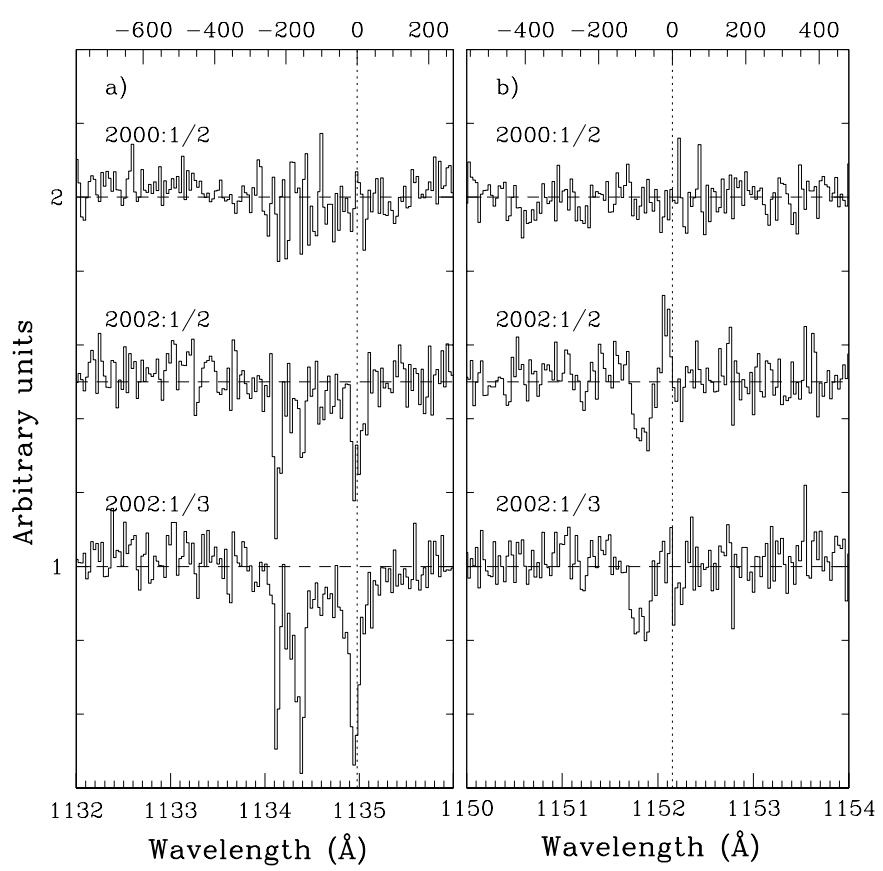

Fig. 9. Short-timescale variations in HD 100546 FUSE spectra. The first sub-exposure is divided by the second one in the 2000 spectrum (top) and by the second and the third respectively in 2002 (middle and bottom). The resulting divided spectra, rebinned by 4 pixels, are plotted in the spectral range of a) the N I triplet and b) the O I $\left({ }^{1} \mathrm{D}\right) \lambda 1152$ line. In both cases, the alignment of the sub-exposures before division can be verified by examination of the ratios in the vicinity of the CS spectral lines.

flux above $1120 \AA$ A prevents the clear identification of any emission lines.

We identified the emission feature at $1035.6 \AA$ as the blue wing of $\mathrm{C}$ II resonance lines. Despite the blending of the
Table 5. Short-timescale variable CS gas in the HD 100546 spectrum of 2002. The velocity of the centroid is given in the stellar rest frame.

\begin{tabular}{|c|c|c|c|}
\hline Ion & $\begin{array}{c}\text { Central vel. } \\
\left(\mathrm{km} \mathrm{s}^{-1}\right)\end{array}$ & $\begin{array}{c}\text { Vel. range } \\
\left(\mathrm{km} \mathrm{s}^{-1}\right)\end{array}$ & $\begin{array}{c}\text { Eq. width } \\
(\mathrm{m} \AA)\end{array}$ \\
\hline \multicolumn{4}{|l|}{ sub-exp1/sub-exp2 } \\
\hline N I $1134.1653 \AA$ & -7.5 & $-40 ;+30$ & 28.6 \\
\hline N I $1134.4149 \AA$ & $"$ & $"$ & 15.4 \\
\hline N I $1134.9803 \AA$ & $"$ & $"$ & 33.4 \\
\hline O I $\left({ }^{1} D\right) 1152.1512 \AA$ & -79.7 & $-135 ;-40$ & 43.0 \\
\hline \multicolumn{4}{|l|}{ sub-exp $1 / s u b-\exp 3$} \\
\hline N I $1134.1653 \AA$ & -9.0 & $-45 ;+40$ & 32.0 \\
\hline N I $1134.4149 \AA$ & $"$ & $"$ & 65.0 \\
\hline N I $1134.9803 \AA$ & $"$ & $"$ & 73.7 \\
\hline O I $\left({ }^{1} \mathrm{D}\right) 1152.1512 \AA$ & -86.2 & $-115 ;-40$ & 47.5 \\
\hline
\end{tabular}

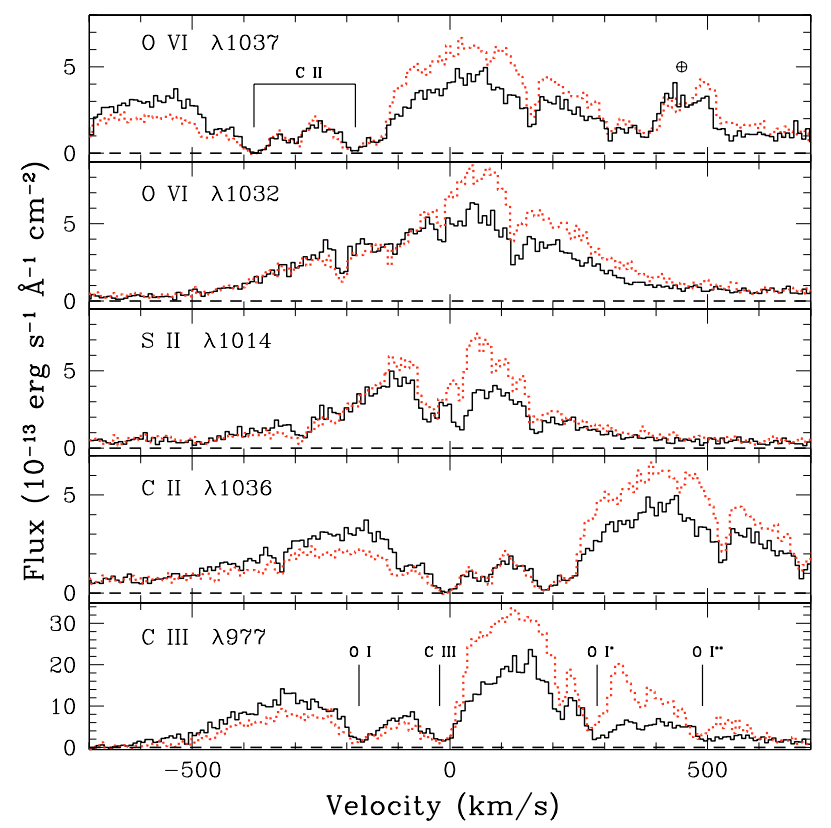

Fig. 10. Emission lines in HD 100546 FUSE spectra (rebinned by 4 pixels), observed on 2000 March 26 (solid line) and 2002 March 3 (dotted line). The airglow line close to the $\lambda 1037 \mathrm{O}$ VI line is labeled with the $\oplus$ symbol. The narrow unlabeled absorption lines arise from molecular hydrogen.

C II resonance doublet with the $\lambda 1037 \mathrm{OVI}$ line, the similarity of this feature to the $\mathrm{C}$ III emission profile and its temporal behavior in the two spectra (see Fig. 10) confirms this interpretation.

All of these emission lines are broad, with a total width of at least $\simeq \pm 400 \mathrm{~km} \mathrm{~s}^{-1}$ and non-Gaussian profiles. Superimposed circumstellar absorption lines, mainly due to $\mathrm{H}_{2}$ electronic transitions (Fig. 10), confuse the line profiles. In addition, all these lines exhibit strong variations between the two epochs.

These lines probe temperatures ranging from $15000 \mathrm{~K}$ up to $5 \times 10^{5} \mathrm{~K}$, and are formed in a dense gaseous medium, 
Table 6. Emission line measurements. The second column gives the total integrated flux of the observed profile (not corrected for reddening). The third column gives the number of components used to model the line profile with simple Gaussians. The last three columns give the parameters for each component: the central velocity in the stellar rest frame, the Full Width Half Maximum $(F W H M)$ and the corresponding integrated flux.

\begin{tabular}{|c|c|c|c|c|c|c|}
\hline Ion & $\begin{array}{cl}\text { Flux } \\
10^{-13}\left(\mathrm{erg} / \mathrm{s} / \mathrm{cm}^{2}\right)\end{array}$ & $\mathrm{Nb}$ & $\begin{array}{r}\text { Center } \\
\left(\mathrm{km} \mathrm{s}^{-1}\right)\end{array}$ & $\begin{array}{l}F W H M \\
\left(\mathrm{~km} \mathrm{~s}^{-1}\right)\end{array}$ & $\begin{array}{c}\text { Flux } \\
10^{-13}\left(\mathrm{erg} / \mathrm{s} / \mathrm{cm}^{2}\right)\end{array}$ & $\begin{array}{c}\text { Ratio } \\
(2002 / 2000)\end{array}$ \\
\hline \multicolumn{7}{|l|}{2000 March 26} \\
\hline \multirow[t]{3}{*}{ C III (977 ̊) } & $28.2 \pm 0.05$ & 3 & -310 & $303 \pm 3$ & $11.88 \pm 0.04$ & \\
\hline & & & +89 & $260 \pm 5$ & $10.94 \pm 1.42$ & \\
\hline & & & +256 & $493 \pm 20$ & $12.53 \pm 1.07$ & \\
\hline C II (1036 ̊̊) & $>2.2$ & $>1$ & $\ldots$ & $\ldots$ & $\ldots$ & \\
\hline \multirow[t]{3}{*}{ O VI (1032 ̊̊) } & $8.4 \pm 0.05$ & 3 & -206 & $270 \pm 10$ & $2.78 \pm 0.17$ & \\
\hline & & & +26 & $215 \pm 18$ & $3.14 \pm 0.40$ & \\
\hline & & & +197 & $275 \pm 40$ & $2.21 \pm 0.53$ & \\
\hline O VI (1037 ̊̊) & $>4.06$ & $\ldots$ & $\ldots$ & $\ldots$ & & \\
\hline \multirow[t]{3}{*}{ S II (1014 Å) } & $4.4 \pm 0.02$ & 3 & -155 & $422 \pm 13$ & $2.10 \pm 0.26$ & \\
\hline & & & -7 & $303 \pm 7$ & $3.29 \pm 0.46$ & \\
\hline & & & +148 & $512 \pm 62$ & $1.15 \pm 0.24$ & \\
\hline \multirow[t]{3}{*}{ S II (1019 ̊) } & $3.4 \pm 0.02$ & 3 & -155 & $408 \pm 20$ & $1.18 \pm 0.12$ & \\
\hline & & & -7 & $270 \pm 9$ & $2.48 \pm 0.11$ & \\
\hline & & & +148 & $430 \pm 22$ & $0.78 \pm 0.12$ & \\
\hline \multicolumn{7}{|l|}{2002 March 3} \\
\hline \multirow[t]{3}{*}{ C III (977 ̊) } & $36.0 \pm 0.06$ & 3 & -308 & $260 \pm 13$ & $7.01 \pm 0.44$ & 0.59 \\
\hline & & & +90 & $207 \pm 27$ & $15.36 \pm 4.90$ & 1.40 \\
\hline & & & +267 & $412 \pm 33$ & $23.63 \pm 4.80$ & 1.88 \\
\hline C II (1036 ̊̊) & $>1.0$ & $>1$ & $\ldots$ & $\ldots$ & $\ldots$ & $>0.45$ \\
\hline \multirow[t]{3}{*}{ O vi (1032 Å) } & $10.9 \pm 0.02$ & 3 & -217 & $264 \pm 14$ & $2.32 \pm 0.18$ & 0.83 \\
\hline & & & +44 & $206 \pm 7$ & $4.13 \pm 0.39$ & 1.32 \\
\hline & & & +184 & $312 \pm 14$ & $3.89 \pm 0.34$ & 1.76 \\
\hline O vi (1037 ̊̊) & $>6.7$ & $\ldots$ & $\ldots$ & $\ldots$ & $\ldots$ & \\
\hline \multirow[t]{3}{*}{ S II (1014 Å) } & $6.1 \pm 0.03$ & 3 & -163 & $424 \pm 53$ & $1.29 \pm 0.13$ & 0.61 \\
\hline & & & +1 & $239 \pm 9$ & $4.75 \pm 0.21$ & 1.44 \\
\hline & & & +98 & $518 \pm 42$ & $2.15 \pm 0.24$ & 1.87 \\
\hline \multirow[t]{3}{*}{ S II (1019 ̊) } & $4.6 \pm 0.02$ & 3 & -163 & $408 \pm 53$ & $0.76 \pm 0.08$ & 0.64 \\
\hline & & & +1 & $201 \pm 7$ & $3.37 \pm 0.19$ & 1.36 \\
\hline & & & +98 & $433 \pm 37$ & $1.38 \pm 0.06$ & 1.77 \\
\hline
\end{tabular}

requiring an additional source of ionizing radiation or collisional heating. The integrated fluxes were measured for each isolated emission line, but not corrected for interstellar extinction (Table 6). Indeed, due to the uncertainty in the parameterization of the reddening curves below $1200 \AA$ and the fact that the bulk the extinction should be due to the CS dust, we choose to analyze the observed spectra. In the case of the O VI doublet lines, we restrict the analysis to the isolated strong line at $1032 \AA$. The blue wing of the $\lambda 1037$ line is affected by the C II emission line and by strong absorption lines due to $\mathrm{H}_{2}$ and $\mathrm{C}$ II.

To investigate the origin of these strong emission lines and the physical process which can explain their observed spectral behavior, we performed a qualitative analysis of the various line profiles and their temporal variations.

\subsection{Emission line profile decomposition}

All the emission lines display a complex profile which can not be modeled by a simple Gaussian. Except for the S II lines, the profiles are not symmetric (see Fig. 11). In addition, comparison of the two epochs shows that the line profiles varied in intensity but presented no significant changes in their global shapes (Fig. 10).

The line profiles were decomposed using a combination of Gaussians to model the line shape and to investigate the 


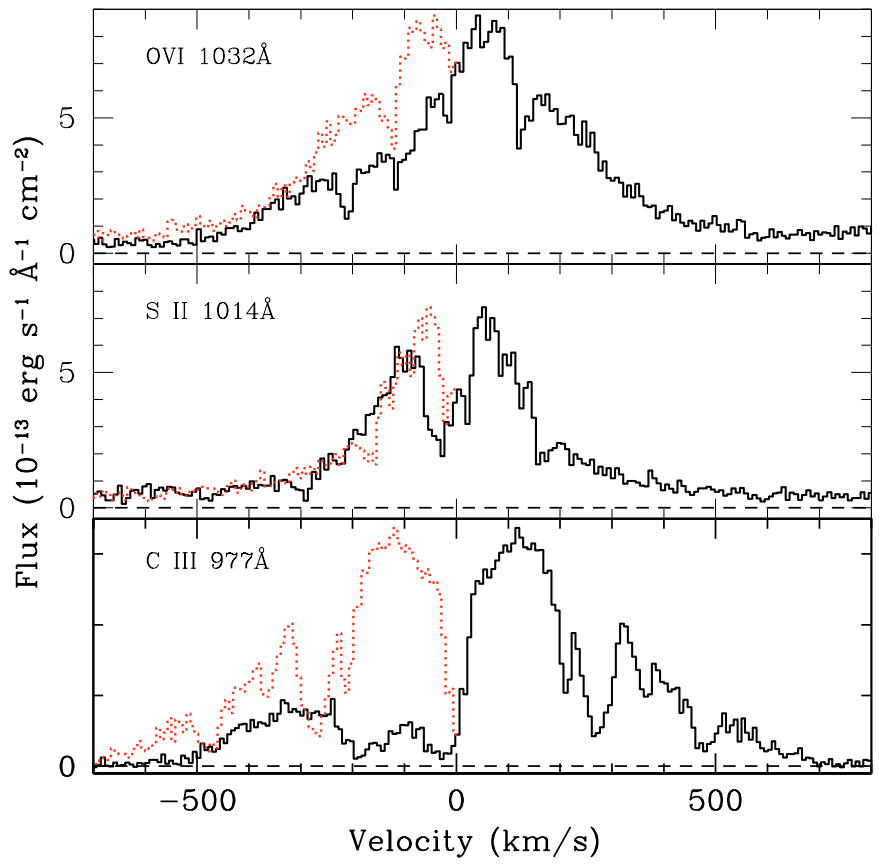

Fig. 11. O VI $\lambda 1032$, S II $\lambda 1014$ and C III $\lambda 977$ emission lines as observed in the 2002 spectra (rebinned by 4 pixels). The dotted line is the red side of the emission line reflected about zero velocity in the stellar rest frame, highlighting the profile asymmetry.

temporal behavior of the different parts of the profiles. This analysis was made using the interactive SPECFIT fitting package, which determined the best fit using a non-linear $\chi^{2}$ minimization statistic (Kriss 1994). Each Gaussian was allowed to vary in intensity, position, and FWHM. In the case of the S II doublet, the positions of the components at $1019 \AA$ were scaled by the doublet ratio value to the $1014 \AA$ ones. We checked that the same decomposition of the profile reproduces the same line observed at the two epochs. The corresponding derived parameters for the emission lines are given in Table 6 .

The C III resonance line presents the broadest and also the most complex profile. It displays a strong peak which is slightly redshifted and two broad components: one blueshifted component clearly separated from the main peak, and an extra redshifted broad component necessary to reproduce the red wing of the profile. The overlying absorption lines are due to $\mathrm{H}_{2}$ and $\mathrm{O}$ I transitions, except for the absorption feature at $976.90 \AA$ that we identify as C III. Here we stress that the same phenomenon is observed in the C II profile: two absorption features from the $\mathrm{C}$ II resonance doublet are superimposed on the C II emission profile. These absorption features are broad, favoring a CS origin, and moderately blueshifted relative to the stellar rest frame (see Table 3).

Three Gaussians are necessary to fit all the emission lines: a central peak with a moderate redshift and two much broader components, blueshifted and redshifted respectively. This structure is obvious in the C III lines, where the bluest component is clearly separated from the main peak. The asymmetry in the profile induced by this blueshifted component is also apparent in the $\lambda 1032 \mathrm{O}$ VI line (see Figs. 11 and 12). On the other hand, the S II excited line profiles appear fairly

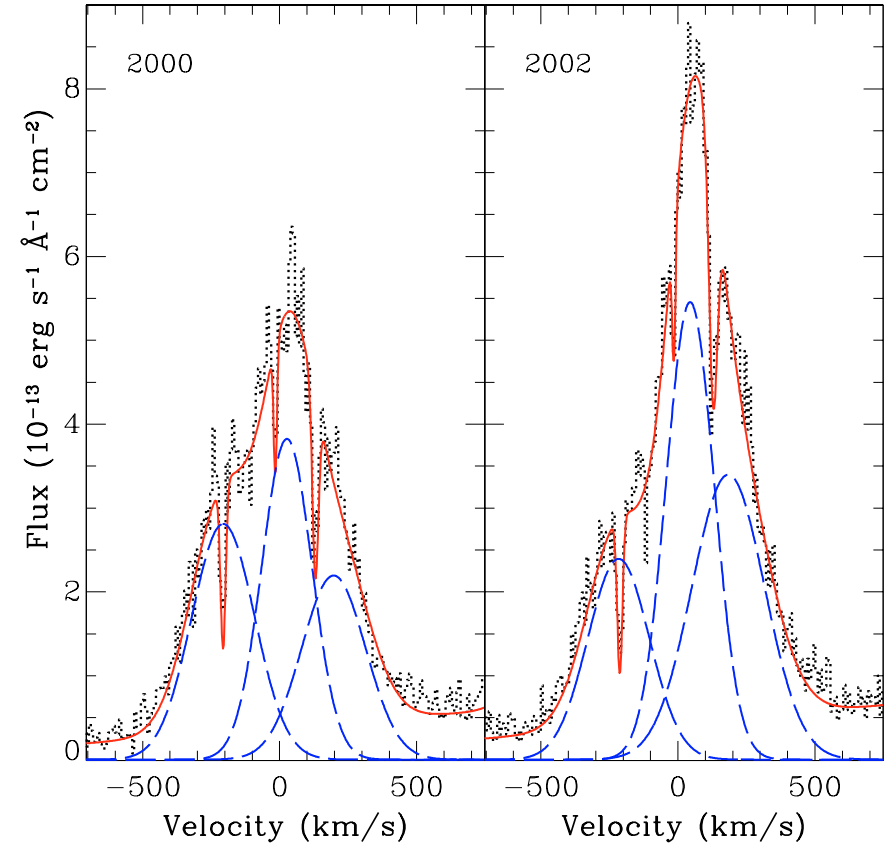

Fig. 12. Example of line profile decomposition for the $\lambda 1032$ O VI emission line observed in 2000 (left) and 2002 (right). The dotted line is the observed profile, rebinned by 4 pixels. The individual fitted components are plotted with dashed lines and the solid line is the resulting profile. Note that we included the $\mathrm{H}_{2}$ absorption lines in the profile fitting.

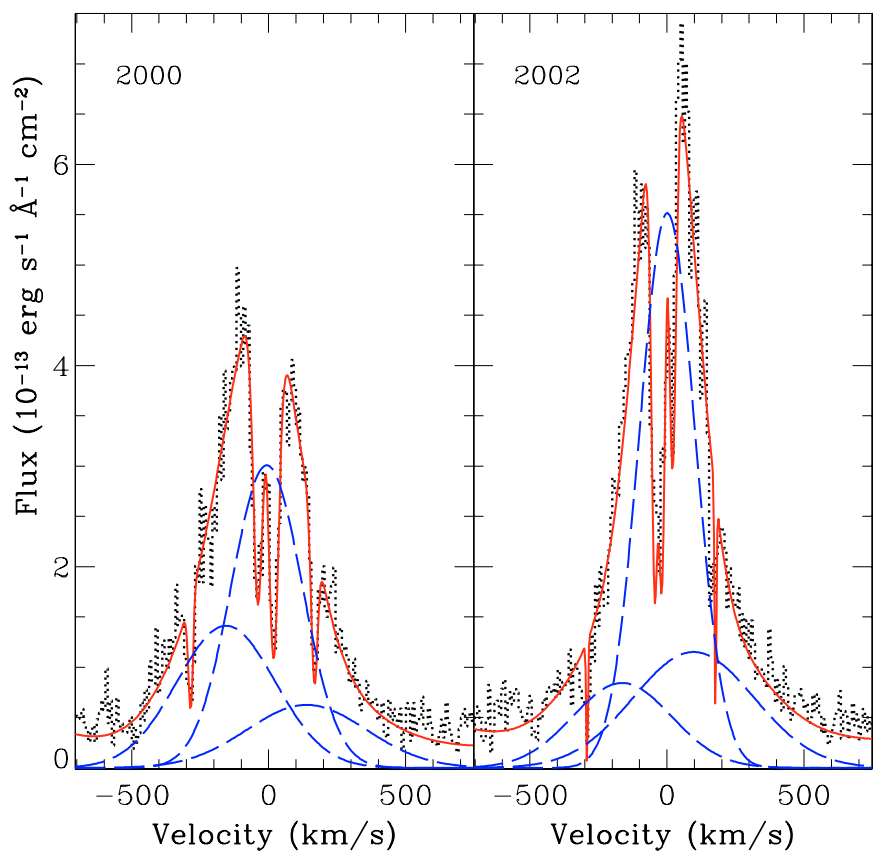

Fig. 13. Same as Fig. 12 but for the S II line at $1014 \AA$ A.

symmetric, especially in 2002. However, the residual obtained with a double-Gaussian fit on the 2000 spectrum highlights the presence of a weak additional blueshifted emission component, which was included in the fit (Fig. 13). Concerning the S II doublet lines, note that the central depression, which is observed in both doublet lines, corresponds to overlying $\mathrm{H}_{2}$ absorption lines (Lecavelier des Etangs et al. 2003) and not to S II. These 
superimposed absorption lines were taken into account in our fitting procedure.

For each line except the C II and the O VI $\lambda 1037$ lines, we compared the ratio of the total integrated observed flux in the two exposures. We found nearly the same average ratio, $\simeq 1.3$ at $7 \%$, showing that the flux variations in all the lines are correlated. The comparison of the ratio of the integrated flux for each fitted component in each emission line (Table 6) confirms this correlation in profile variation. As a consequence, even if the multiple-component decomposition of the emission lines indicates the presence of different processes, the correlation in time-variability points to a common formation mechanism for these emission lines. On the other hand, despite this similar structure, we found a spread in velocities and widths from one ion to another, which shows that these species do not form at the same location within the same region.

In addition, as evidenced by the C III and C II lines (Fig. 10), the variation of the bluest component in all emission lines is clearly anti-correlated with the redshifted component. This confirms that the adopted three-Gaussian decomposition is relevant and shows that the time evolution of the different processes leading to the formation of the different components should be linked.

Finally, in both spectra, the observed line ratio of the S II doublet is not in agreement with the oscillator strength ratio, as the $1014 \AA$ line is expected to be 1.81 that of the $1019 \AA$ line. The profile decomposition shows however that while the bluest component is close to the expected ratio in both spectra, the central and the reddest ones are not. These components appear related to an optically thick medium. A similar result is suspected for the $\mathrm{O}$ VI doublet lines, but for this element, the blending of the $\lambda 1037$ line with the $\mathrm{C}$ II line prevents detailed analysis.

\section{Discussion}

\subsection{Summary}

The results can be summarized as follows:

- Strong emission lines with large line width are detected from ionized species sampling a wide range in temperature. All the observed line profiles can be decomposed with three components, suggesting a similar composite origin. Neither a thermal effect nor stellar rotation can account for the large width of the lines.

- All the emission lines vary substantially on a timescale of years. The profiles do not change significantly in shape but rather in strength. The variations in the integrated fluxes are of the same amplitude between the spectra taken two years apart. But for all species, the blueshifted component is weaker in 2000 than in 2002, while the two other components are stronger. This suggests an anti-correlation in the variation and a link between the processes which form the different parts of the profiles.

- For the S II and O VI doublets, the ratios of the two doublet lines indicate different physical conditions in the line formation regions.
Table 7. CS absorption lines identified in the HD 100546 FUSE spectrum. Below $1100 \AA$, the low flux level does not allow clear identification of weak CS atomic lines, and the main CS features in the spectrum are due to $\mathrm{H}_{2}$ transitions.

\begin{tabular}{|c|c|c|}
\hline $\begin{array}{l}\text { Wavelength } \\
(\AA)\end{array}$ & Ion & $\begin{array}{l}\text { Energy } \\
\left(\mathrm{cm}^{-1}\right)\end{array}$ \\
\hline 976.448 & O I & 0.000 \\
\hline 977.020 & C III & 0.00 \\
\hline 977.959 & O I & 158.26 \\
\hline 978.617 & O I & 226.977 \\
\hline 1036.336 & $\mathrm{C}_{\text {II }}$ & 0.000 \\
\hline 1037.017 & C II & 63.43 \\
\hline 1048.219 & ArI & 0.00 \\
\hline 1063.176 & Fe II & 0.00 \\
\hline 1066.659 & ArI & 0.00 \\
\hline 1067.614 & $\mathrm{NI}^{2} \mathrm{D}$ & 19224.46 \\
\hline 1068.346 & Fe II & 384.79 \\
\hline 1068.611 & $\mathrm{NI}^{2} \mathrm{D}$ & 19223.18 \\
\hline 1081.875 & Fe II & 0.00 \\
\hline 1083.993 & N II & 0.00 \\
\hline 1084.565 & $\mathrm{~N}_{\text {II }}$ & 48.67 \\
\hline 1084.58 & N II & 48.67 \\
\hline 1085.532 & N II & 130.80 \\
\hline 1085.551 & N II & 130.80 \\
\hline 1085.709 & N II & 130.80 \\
\hline 1096.607 & Fe II & 384.79 \\
\hline 1096.877 & Fe II & 0.00 \\
\hline 1125.448 & Fe II & 0.00 \\
\hline 1126.840 & Fe II & 384.79 \\
\hline 1126.955 & Fe II & 384.79 \\
\hline 1126.421 & Fe II & 667.68 \\
\hline 1133.665 & Fe II & 0.00 \\
\hline 1134.165 & N I & 0.00 \\
\hline 1134.414 & $\mathrm{~N}_{\mathrm{I}}$ & 0.00 \\
\hline 1134.165 & $\mathrm{NI}_{\mathrm{I}}$ & 0.00 \\
\hline 1142.366 & Fe II & 0.00 \\
\hline 1143.226 & Fe II & 0.00 \\
\hline 1143.466 & $\mathrm{NI}^{2} \mathrm{P}$ & 28838.92 \\
\hline 1143.651 & $\mathrm{NI}^{2} \mathrm{P}$ & 28839.31 \\
\hline 1144.938 & Fe II & 0.00 \\
\hline 1147.409 & Fe II & 384.79 \\
\hline 1148.079 & Fe II & 862.61 \\
\hline 1148.277 & Fe II & 384.79 \\
\hline 1149.958 & PII & 164.90 \\
\hline 1150.685 & Fe II & 667.68 \\
\hline 1151.146 & Fe II & 667.68 \\
\hline 1152.151 & O I ${ }^{1} \mathrm{D}$ & 15867.86 \\
\hline 1152.818 & P II & 0.00 \\
\hline 1152.818 & P II & 0.00 \\
\hline 1152.875 & Fe II & 862.61 \\
\hline 1153.272 & Fe II & 862.61 \\
\hline 1153.995 & P II & 469.12 \\
\hline 1155.013 & P II & 164.90 \\
\hline 1159.816 & $\mathrm{NI}_{\mathrm{I}}$ & 0.00 \\
\hline 1160.936 & $\mathrm{~N}_{\mathrm{I}}$ & 0.00 \\
\hline 1162.015 & Mn II & 0.00 \\
\hline 1163.326 & Mn II & 0.00 \\
\hline 1163.883 & $\mathrm{NI}_{\mathrm{I}}$ & 19224.46 \\
\hline 1164.208 & Mn II & 0.00 \\
\hline 1164.324 & $\mathrm{NI}^{2} \mathrm{D}$ & 19233.18 \\
\hline 1167.448 & $\mathrm{NI}^{2} \mathrm{D}$ & 19224.46 \\
\hline 1168.535 & $\mathrm{NI}^{2} \mathrm{D}$ & 19233.18 \\
\hline 1176.509 & $\mathrm{NI}^{2} \mathrm{D}$ & 19224.46 \\
\hline 1177.694 & $\mathrm{NI}^{2} \mathrm{D}$ & 19233.18 \\
\hline
\end{tabular}


- Broad blueshifted absorption features are detected in C II and C III, with a shift of the velocity centroid of a few $\mathrm{km} \mathrm{s}^{-1}$ to the blue in 2002 .

- Temporal variations of the continuum flux level are observed. They need however to be confirmed.

- Some absorption lines exhibit a high level of variability on various timescales. Variations are clearly detected in the NI lines from the ground state and from $\left({ }^{2} \mathrm{D}\right)$ and $\left({ }^{2} \mathrm{P}\right)$ metastable levels, as well as in the OI resonance and ${ }^{1} \mathrm{D} \lambda 1152$ lines. They appear as broad extra absorption in the 2000 spectrum, with velocity extent from -200 to $+320 \mathrm{~km} \mathrm{~s}^{-1}$ relative to the star's radial velocity. A similar behavior is marginally detected in the strongest Fe II resonance lines.

- Ar I and N II resonance absorption lines are blueshifted and show time variation, with an additional emission feature occurring in 2002. Both ions appear related to an outflow.

- Short-term variations with low velocity extent are detected only in the blue wing of the N I resonance triplet and the O I ${ }^{1} \mathrm{D}$ lines. Such variations are highly sporadic, as this phenomenon was not observed in 2000.

\subsection{Discussion}

As previously pointed out, variations of HD 100546 have been reported in photopolarimetic (van den Ancker et al. 1998; Clarke et al. 1999) and in spectroscopic observations (Grady et al. 1996; Vieira et al. 1999). In particular, in a study carried out on a set of IUE spectra, Grady et al. (1997) reported accreting gas profiles observed in various volatile and mildly refractory absorption lines such as C I, Mg II, Fe II. They interpreted these transient spectral signatures as originating in the comae of star-grazing bodies like comets or asteroids, by analogy with the Falling Evaporating Bodies (FEB) model developed for the $\beta$ Pictoris system (see Lagrange et al. 2000, and references therein). However, Beust et al. (2001) brought this interpretation into question for Herbig Ae/Be stars. Even though Beust et al. (2001) concluded that this scenario could not be totally excluded in the case of the HD 100546 system, the presence of a structured wind as inferred by Vieira et al. (1999) should prevent the observation of FEBs spectral signatures.

In our case, the $\beta$ values, which are the ratios of the radiation pressure from the central star to the stellar gravity (see Lagrange et al. 1996), estimated from our FUSE data for each variable species (see Table 4), are compatible with this interpretation. Indeed, even for N I and O I, which are less sensitive to the stellar pressure than Fe II, $\beta$ is greater than 1 , which shows that the gas should be blueshifted and blown away from the star. Such an effect may explain the long and short-term blueshifted variable components observed. On the other hand, the high velocities of the redshifted additional absorption components are close to the free-fall velocity, implying an origin from parent bodies very close to the star. In that case, according to Beust et al. (2001), one should observe spectral variations in metallic ions, as refractory grains are expected to evaporate in the immediate vicinity of the star. The gas should be thus depleted in volatiles which is in contradiction with the present observations.

Besides, as routinely observed in HAeBes, Vieira et al. (1999) reported the presence of strong, broad, and variable $\mathrm{H} \alpha$, $\mathrm{H} \beta$ and He I $5876 \AA$ emission lines in the HD 100546 system but which are not observed in $\beta$ Pictoris. In particular, the $\mathrm{H} \alpha$ line shows a double-peaked profile with changes in relative intensity between the blue and the red peak on timescales of hours to months and typical velocity extent larger than $\pm 400 \mathrm{~km} \mathrm{~s}^{-1}$. The authors concluded that active mass exchange between the CS disk and the star takes place in the close CS environment through accretion and stellar wind. It is worth noting that the main characteristics of these visible emission lines (large width, variability and multiple components in the profile) resemble those of the FUV emission lines. Despite the long time lag between the visible observations and the FUSE ones, the similarity of their emission line characteristics suggests a connection between the line formation processes.

Among pre-main sequence stars, the young classical $\mathrm{T}$ Tauri stars (CTTs) present similar spectral characteristics. They commonly exhibit strong emission lines in neutral and ionized states of various species, with large line width and substantial variability in their line profiles (e.g. Alencar \& Basri 2000). Together with other characteristics, such as excess continuum flux from the UV to the near-IR, these characteristics are usually interpreted as signs of magnetospheric accretion (see Najita et al. 2000). In such a model, matter from the disrupted disk moves away from the disk plane and flows inward along the stellar magnetic field lines, reaching the stellar surface with a velocity close to the free-fall velocity (Hartmann et al. 1994; Muzerolle et al. 1998, 2001). For several premain sequence stars, like TW Hya (Alencar \& Batalha 2002), DR Tau (Alencar et al. 2001), and AA Tau (Bouvier et al. 2003), this model explains the observed characteristics well.

\subsection{The magnetospheric accretion hypothesis}

Formation of highly ionized species requires high densities. Classically, in solar-like main sequence stars (see Ayres 2000) such species form in a chromosphere/transition region above the stellar photosphere. In the specific case of Herbig Ae/Be stars, the existence of extended chromospheres is now established (e.g. Boehm \& Catala 1995; Bouret \& Catala 2000). Even though the central emission component observed in the HD 100546 FUV emission lines could originate in such a region, the full complex non-symmetric profile, as observed in the $\mathrm{C} \mathrm{III} \mathrm{and} \mathrm{O}$ VI lines, cannot. It appears thus difficult to form these emission lines solely in a chromospheric region.

On the other hand, the models developed for CTTs can account for complex profiles. In addition, they can also explain the presence of ionized metals. In this model, accreting gas is channeled along magnetic field lines and falls onto the surface of the star at velocities close the free-fall velocity. As a direct consequence of the supersonic velocities in the infalling stream, a shock forms close the stellar surface (Calvet \& Gullbring 1998; Gullbring et al. 2000). The gas can be heated 
up to temperatures of a few $10^{6} \mathrm{~K}$; the kinetic energy is dissipated, producing excess UV emission. No complete theoretical investigations have been carried out studying the metallic ions which can be formed, but UV observations of CTTs commonly show broad C IV and Si IV emission lines (e.g. Errico et al. 2000, 2001; Ardila et al. 2002). For these species, JohnsKrull et al. (2000) and Ardila et al. (2002) have shown that a large fraction of the emission does come from processes related to accretion.

Adopting the idea of magnetospheric accretion in HD 100546, the central peak and the slightly redshifted infalling gas can be produced in the accretion flow. More precisely, the ions should be formed within the accretion shock, close to the stellar surface, in regions where the temperatures are the highest and the velocities closest to free-fall. In that case, the large width of the lines could simply result from turbulence, as the gas is expected to achieve supersonic velocities and high density, as suggested by the line ratio of the doublet lines. Since the inclination of the HD 100546 system is $50^{\circ}$ relative to the line of sight and assuming that the disk is disrupted at a few stellar radii by the magnetic field (Hartmann et al. 1994), the lines from the hot gas should probe the polar region and the inner region of the system.

The observed variations in the N I and OI absorption lines could also be caused by magnetospheric accretion processes. According to Martin (1996), neutral gas should also follow the magnetic field lines, due to collisional coupling between ions and neutrals. Because of their low ionization potential, neutral species originate in regions of much lower temperature and density, probably close to the disk, as suggested by Muzerolle et al. (2001) for the more fragile ion $\mathrm{NaI}$. In that picture, the accreting gas could explain the additional redshifted absorption observed in the 2000 spectrum, as well as the slightly blueshifted extra emission, which could be due to very weak emission occurring in the 2002 data.

The similarities observed in the temporal behavior of neutral species favors a common origin and suggests that the same process affects them. On the other hand, they are clearly not related to the same region of the magnetosphere as the emission lines and furthermore, their variations are probably not directly related to each other.

The presence of the broad blueshifted emission component, as well as the blueshifted absorption features observed in C II and C III, suggest a strong contribution from a hot wind whose projected size does not cover the full stellar disk, at least in C III. The quasi-symmetry in velocity derived for the bluest and reddest fitted components and the anti-correlation in their variations show that they are consistent with a variable double-peaked profile. Such a profile can be obtained in an envelope rotating at several times the star's velocity in its inner part and expanding in its outer regions (Mihalas \& Conti 1980). The terminal velocity, measured from the blue wing of the profile, is $-620 \mathrm{~km} \mathrm{~s}^{-1}$ for C III and $-550 \mathrm{~km} \mathrm{~s}^{-1}$ for C II, with no change between the two spectra. This hot wind could thus emerge along the open field lines in the polar region of the star. The inclination of HD 100546 system would produce the observed asymmetry between the two peaks (Petrenz \& Puls 1996). In addition, as suggested by the ratio of the integrated flux in the emission lines between the two spectra, the outflow and accretion processes are related in this system. The likely variations in the accretion rate should thus translate into a modulation of the outflow.

The spectral variations observed in Ar I, N II, and also the blue extent of the additional absorption in the N I and O I lines in 2002 are also consistent with a wind contribution to the profile. However, whether all these lines arise in a single region or also from the magnetosphere/disk boundary remains an open question.

The main underlying assumption of such an interpretation is that these active phenomena are related to a magnetic field. The existence of magnetic fields in Herbig Ae/Be stars remains the subject of controversy, since according to standard stellar theory, these intermediate mass stars are not supposed to posses sub-photospheric convective zones necessary to trigger dynamo effects. However, whatever its origin, evidence for the presence of large-scale magnetic fields in HAeBe stars is accumulating (Boehm \& Catala 1995). But the direct detection of magnetic fields remains difficult, especially if the intensity is only about a few hundred Gauss. For HD 100546, no detection of a magnetic field has been reported (Donati et al. 1997). However, the authors did not rule out the possibility that the star could have a magnetic field whose intensity would require much higher sensitivity to be detected.

In any case, it is clear that our data support the presence of a stellar magnetosphere interacting with a CS disk. As stressed by Corcoran \& Ray (1998), this reinforces the idea of a common mechanism operating among the CTTs and HAeBes with disk that is responsible for activity.

\section{Conclusion}

In this paper, we presented and analysed in detail the first FUV spectra of HD 100546, one of the first Herbig Be stars for which the presence of a CS disk has been clearly established. Besides numerous absorption lines, mainly due to volatile elements, strong emission lines are clearly detected above the very low stellar flux level below $1140 \AA$. These lines are resonance transitions of highly ionized species (C III and O VI), as well as transitions from excited levels in S II, showing the presence of very high temperature regions. They span a wide range in excitation and in ionization potential, and provide evidence for the presence of a source of heating in addition to the radiative flux from the photosphere.

Spectral variations are observed on timescales from one hour to years. Short-timescale variations are limited to the N I and $\mathrm{OI}$ absorption lines and are related to outflow events. Long-timescale variations affect not only CS absorption lines but also emission lines and the photospheric flux. These spectral features are related to both accretion and mass loss, consistent with results obtained from visible observations by Vieira et al. (1999) of Balmer and He I lines. Analysis of the variations supports the idea that accretion and outflow are connected in this system.

Two different scenarios have been discussed for the origin of this variable gas: evaporation from star-grazing bodies and magnetospheric accretion. Although we cannot rule out the 
possibility that at least part of the spectral variations observed in N I, O I, and marginally in Fe II absorption lines could be the signature of infalling evaporating bodies, this scenario has difficulty explaining the depth and the large velocity extent of the observed features, as well as the lack of any significant metal enhancement in the infalling gas. Furthermore, as previously discussed in the case of $\beta$ Pictoris (Deleuil et al. 2001), another mechanism is required to explain the emission lines.

The second interpretation involves the presence of a stellar magnetosphere, likely interacting with the innermost part of the CS disk, and a stellar wind. By analogy with the magnetospheric accretion models proposed for classical $\mathrm{T}$ Tauri stars, we suggest that the emission lines originate in a magnetospheric accretion flow. The energy dissipated when the flow reaches the stellar surface may be sufficient to create highly ionized species, such as O VI, whose presence could not be accounted for by simple photoionization. One of the main advantages of this scenario is that it can account for the main features observed in the spectrum of HD 100546: emission lines and spectral variations.

This interpretation needs to be further explored from both the theoretical and observational sides. Spectroscopic monitoring of the star, with simultaneous observations in the visible and in the FUV and high time sampling, is necessary to investigate the dynamical evolution of and connection between the different processes, and to constrain the geometry of the system.

Acknowledgements. This work is based on data obtained for the Guaranteed Time Team by the NASA-CNES-CSA FUSE mission operated by the Johns Hopkins University. Financial support to US participants has been provided by NASA contract NAS5-32985. This work has used the profile fitting procedure Owens.f developed by M. Lemoine and the French FUSE Team. JCB acknowledges the CNES for financial support. We thank M. André and J. M. Désert for processing of the data.

\section{References}

Abt, H. A., Levato, H., \& Grosso, M. 2002, ApJ, 573, 359

Alencar, S. H. P., \& Basri, G. 2000, AJ, 119, 1881

Alencar, S. H. P., \& Batalha, C. 2002, ApJ, 571, 378

Alencar, S. H. P., Johns-Krull, C. M., \& Basri, G. 2001, AJ, 122, 3335

Ardila, D. R., Basri, G., Walter, F. M., Valenti, J. A., \& Johns-Krull, C. M. 2002, ApJ, 566, 1100

Augereau, J. C., Lagrange, A. M., Mouillet, D., \& Ménard, F. 2001, A\&A, 365, 78

Aumann, H. H., Beichman, C. A., Gillett, F. C., et al. 1984, ApJ, 278, L23

Ayres, T. R. 2000, Sol. Phys., 193, 273

Barstow, M. A., Good, S. A., Holberg, J. B., et al. 2002, MNRAS, 330, 425

Beust, H., Karmann, C., \& Lagrange, A.-M. 2001, A\&A, 366, 945

Boehm, T., \& Catala, C. 1995, A\&A, 301, 155

Bouret, J.-C., \& Catala, C. 2000, A\&A, 359, 1011

Bouret, J.-C., Martin, C., Deleuil, M., Simon, T., \& Catala, C. 2003, A\&A, 410, 175

Bouvier, J., Grankin, K., Alencar, S., et al. 2003, A\&A, 409, 169

Calvet, N., \& Gullbring, E. 1998, ApJ, 509, 802
Catala, C., Bohm, T., Donati, J.-F., \& Semel, M. 1993, A\&A, 278, 187

Chiang, E. I., Joung, M. K., Creech-Eakman, M. J., et al. 2001, ApJ, 547,1077

Clarke, D., Smith, R. A., \& Yudin, R. V. 1999, A\&A, 347, 590

Corcoran, M., \& Ray, T. P. 1998, A\&A, 331, 147

Deleuil, M., Bouret, J.-C., Lecavelier des Etangs, A., et al. 2001, ApJ, 557, L67

Donati, J.-F., Semel, M., Carter, B. D., Rees, D. E., \& Collier Cameron, A. 1997, MNRAS, 291, 658

Errico, L., Lamzin, S. A., \& Vittone, A. A. 2000, A\&A, 357, 951

Errico, L., Lamzin, S. A., \& Vittone, A. A. 2001, A\&A, 377, 557

Gerbaldi, M., Faraggiana, R., \& Balin, N. 2001, A\&A, 379, 162

Grady, C. A., Devine, D., Woodgate, B., et al. 2000, ApJ, 544, 895

Grady, C. A., Perez, M. R., Talavera, A., et al. 1996, A\&AS, 120, 157

Grady, C. A., Polomski, E. F., Henning, T., et al. 2001, AJ, 122, 3396

Grady, C. A., Sitko, M. L., Bjorkman, K. S., et al. 1997, ApJ, 483, 449

Gullbring, E., Calvet, N., Muzerolle, J., \& Hartmann, L. 2000, ApJ, 544,927

Hartmann, L., Hewett, R., \& Calvet, N. 1994, ApJ, 426, 669

Hillenbrand, L. A., Strom, S. E., Vrba, F. J., \& Keene, J. 1992, ApJ, 397,613

Johns-Krull, C. M., Valenti, J. A., \& Linsky, J. L. 2000, ApJ, 539, 815

Kriss, G. 1994, in Astronomical Data Analysis Software and Systems III, ASP Conf. Ser., 61, 437

Lagrange, A.-M., Backman, D. E., \& Artymowicz, P. 2000, Protostars and Planets IV, 639

Lagrange, A.-M., Plazy, F., Beust, H., et al. 1996, A\&A, 310, 547

Lecavelier des Etangs, A., Deleuil, M., Vidal-Madjar, A., et al. 2003, A\&A, 407, 935

Lemoine, M., Vidal-Madjar, A., Hébrard, G., et al. 2002, ApJS, 140, 67

Malfait, K., Waelkens, C., Waters, L. B. F. M., et al. 1998, A\&A, 332, L25

Martin, S. C. 1996, ApJ, 470, 537

Mihalas, D., \& Conti, P. S. 1980, ApJ, 235, 515

Miroshnichenko, A., Ivezić, Ž., Vinković, D., \& Elitzur, M. 1999, ApJ, 520, L115

Moos, H. W., Cash, W. C., Cowie, L. L., et al. 2000, ApJ, 538, L1

Morton, D. C. 2000, ApJS, 130, 403

Muzerolle, J., Calvet, N., \& Hartmann, L. 1998, ApJ, 492, 743

Muzerolle, J., Calvet, N., \& Hartmann, L. 2001, ApJ, 550, 944

Najita, J. R., Edwards, S., Basri, G., \& Carr, J. 2000, Protostars and Planets IV, 457

Pantin, E., Waelkens, C., \& Lagage, P. O. 2000, A\&A, 361, L9

Petrenz, P., \& Puls, J. 1996, A\&A, 312, 195

Pezzuto, S., Strafella, F., \& Lorenzetti, D. 1997, ApJ, 485, 290

Roberge, A., Lecavelier des Etangs, A., Grady, C. A., et al. 2001, ApJ, 551, L97

Royer, F., Grenier, S., Baylac, M.-O., Gómez, A. E., \& Zorec, J. 2002, A\&A, 393, 897

Sahnow, D. J., Moos, H. W., Ake, T. B., et al. 2000, ApJ, 538, L7

Smith, B. A., \& Terrile, R. J. 1984, Science, 226, 1421

The, P. S., de Winter, D., \& Perez, M. R. 1994, A\&AS, 104, 315

van den Ancker, M. E., de Winter, D., \& Tjin A Djie, H. R. E. 1998, A\&A, 330, 145

Vidal-Madjar, A., Lecavelier des Etangs, A., \& Ferlet, R. 1998, Planet. Space Sci., 46, 629

Vieira, S. L. A., Pogodin, M. A., \& Franco, G. A. P. 1999, A\&A, 345, 559

Waelkens, C., Waters, L. B. F. M., de Graauw, M. S., et al. 1996, A\&A, 315, L245

Yudin, R. V. 2000, A\&AS, 144, 285 